\title{
A RIEMANN PROBLEM FOR AN ELASTIC BAR THAT CHANGES PHASE
}

By

\author{
YIER LIN
}

Department of Mechanical Engineering, Massachusetts Institute of Technology, Cambridge, Massachusetts

\begin{abstract}
This paper is concerned with the dynamics of an elastic bar that can undergo reversible stress-induced phase transformations. We consider a Riemann problem in which the initial strains belong to a single metastable phase and prove uniqueness of solution that satisfies a nucleation criterion and a kinetic law at all subsonic and sonic phase boundaries. This paper generalizes the results of [3]; the authors of [3] considered a piecewise-linear material for which no wave fans exist, shock waves always travel at the acoustic speed, and shock waves are dissipation-free. The material model of the present paper does not suffer from these degeneracies.
\end{abstract}

1. Introduction. In the simplest one-dimensional theory describing the longitudinal motions of an elastic bar, one employs a pair of conservation laws associated with momentum balance and kinematic compatibility. When the motion of the bar involves a propagating strain discontinuity, it is subject to a pair of jump conditions associated with these conservation laws. In addition, the second law of thermodynamics requires that the dissipation associated with the moving discontinuity be nonnegative, a condition usually referred to as the entropy inequality.

The character of the material of the bar enters the conservation laws and jump conditions through the stress-strain relation $\sigma=\hat{\sigma}(\gamma)$. If the material of the bar is such that stress is a monotonically increasing function of strain that is strictly convex or strictly concave, then phase transformations cannot occur, and all propagating discontinuities are shock waves. For a bar made of such a material, it follows from a result of Oleinik [14] that the Cauchy problem for the associated field equations and jump conditions has at most one piecewise-smooth solution that fulfills the entropy inequality; see [13] for a discussion of Oleinik's theorem and related results.

When the material can undergo a reversible, or thermoelastic, phase transformation, the one-dimensional elastic continuum can be characterized by a nonmonotonic relation $\sigma=\hat{\sigma}(\gamma)$ between stress and strain, or equivalently by a nonconvex elastic potential $W(\gamma)$. Typically one encounters a stress response function $\hat{\sigma}(\gamma)$ in which stress first increases with increasing strain, then decreases, and finally increases again;

Received March 31, 1993.

1991 Mathematics Subject Classification. Primary 73, 35.

(C)1995 Brown University 
for example, Ericksen [10]. The rising branches of such a stress-strain curve are identified with different phases of the material, while the declining branch is associated with an "unstable phase". For suitable values of stress, the associated potential energy $G(\gamma, \sigma)=W(\gamma)-\sigma \gamma$ has multiple energy-wells, each energy-well being associated with a distinct phase of the material. During a typical thermomechanical process, the material often moves from one energy-well to another or, equivalently, from one branch of the stress-strain curve to another.

When the stress-strain curve is nonmonotonic and undergoes a change in the sign of its curvature, the Cauchy problem need no longer have a unique solution, even with the entropy inequality in force; see the remarks of Dafermos [9]. In order to secure uniqueness, many researchers have replaced the entropy inequality with various "admissibility conditions" which are to be satisfied by the weak solutions. For example, two different notions of maximum entropy production have been proposed by Dafermos [8], and augmenting the elastic theory with viscosity and capillarity effects has been proposed by Slemrod [15] and Truskinovsky [17]. The implications of these criteria for dynamic phase transitions have been examined by, for example, Hattori [11], James [12], and Shearer [16].

A completely different approach has been proposed and studied in [2-5] based on the observation in [1] that lack of uniqueness arises not only in dynamic motions, but in quasi-static motions as well. It was suggested in [2] that, in addition to the usual constitutive law between stress and strain, further material description in the form of a nucleation criterion and a kinetic relation pertaining to the phase transitions is needed. The importance of a nucleation criterion and a kinetic relation in the description of phase transitions in solids has long been recognized in the materials science literature, e.g., Christian [7].

It was shown in [2] that the inclusion in the continuum theory of the nucleation criterion and the kinetic relation leads to a determinate quasi-static theory whose predictions are in qualitative accord with experiments on shape memory alloys that involve slowly-propagating phase boundaries. A similar result in the dynamical setting for the Riemann problem for a special piecewise-linear elastic material was established in [3]. It was shown in [4, 5] that the maximum entropy rate admissibility criterion and the viscosity-capillarity admissibility criterion may in fact be viewed as being two particular examples of kinetic relations.

The study in [3] was restricted to a piecewise-linear elastic material. The nature of this trilinear material model leads to a considerable simplification in the analysis. First, this material does not sustain wave fans. Second, shock waves always travel at the sound speed. Third, shock waves are dissipation-free. For these reasons, it is natural to question whether the results found in [3] were special to the trilinear material and inquire whether its conclusions hold for more general rising-fallingrising stress-strain curves. This is the objective of the present paper. We show for a material whose rising-falling-rising stress-strain curve is smooth and has a single inflection point, that the nucleation criterion and the kinetic relation (applied to all subsonic and sonic phase boundaries) serve to single out a unique solution to the Riemann problem with initial data in a single, metastable phase. If the kinetic law is 
applied only to phase boundaries that are subsonic and not to those that are sonic, we do not have uniqueness of solution for all initial data. Truskinovsky [18] takes the view that the kinetic relation should not be applied to sonic phase boundaries and that the accompanying nonuniqueness is an instability.

After dealing with various preliminary issues in Secs. 2-4 we turn, in Sec. 5, to deduce the general solution forms to the Riemann problem that are consistent with the entropy inequality. In Sec. 6 we explicitly construct all solutions to the Riemann problem corresponding to initial data with strains in the low-stain phase; the results are summarized, and the nature of the associated nonuniqueness characterized, in Sec. 7. Finally, in Sec. 8 we introduce the nucleation criterion and the kinetic relation, thus leading to uniqueness.

2. Background. Consider longitudinal motions of an elastic bar that is regarded as a one-dimensional continuum with unit cross-sectional area. The motion of the bar is assumed to take place isothermally. During such a motion, the particle at $x$ in the reference configuration is carried to $x+u(x, t)$ at time $t$, where $u(x, t)$ is the displacement. The displacement is assumed to be continuous with piecewisecontinuous first and second derivatives throughout the regions of space-time to be considered. The strain and particle velocity are defined by $\gamma=u_{x}$ and $v=u_{t}$ at points $(x, t)$ where the derivatives exist. Necessarily, $\gamma(x, t)>-1$ in order to ensure that the mapping $x \rightarrow x+u(x, t)$ is invertible at each instant $t$. The stress $\sigma(x, t)$ is related to the strain through

$$
\sigma=\hat{\sigma}(\gamma),
$$

where $\hat{\sigma}$ is the stress response function of the material. At points where $\gamma$ and $v$ are smooth, balance of momentum and kinematic compatibility require that

$$
\hat{\sigma}^{\prime}(\gamma) \gamma_{x}=\rho v_{t}, \quad v_{x}=\gamma_{t},
$$

where the constant $\rho$ is the mass density in the reference configuration. If there is a moving discontinuity at $x=s(t)$, the following jump conditions must hold:

$$
\hat{\sigma}(\stackrel{+}{\gamma})-\hat{\sigma}(\bar{\gamma})=-\rho \dot{s}(\stackrel{+}{v}-\bar{v}), \quad(\stackrel{+}{v}-\bar{v})=-\dot{s}(\dot{\gamma}-\bar{\gamma}),
$$

where for any function $g(x, t)$ we write $\stackrel{ \pm}{g}=g(s(t) \pm, t)$ for the limiting values of $g$ on either side of the discontinuity.

Consider the motion of the piece $x_{1} \leq x \leq x_{2}$ of the bar during a time interval $\left[t_{1}, t_{2}\right]$. Suppose that $\gamma$ and $v$ are smooth on $\left[x_{1}, x_{2}\right] \times\left[t_{1}, t_{2}\right]$ except at the moving discontinuity $x=s(t)$. Let $E(t)$ be the total mechanical energy at time $t$ associated with this piece of bar:

$$
E(t)=\int_{x_{1}}^{x_{2}}\left[W(\gamma(x, t))+\frac{1}{2} \rho v^{2}(x, t)\right] d x,
$$

where $W(\gamma)$ is the strain energy per unit reference volume of the bar, i.e.,

$$
W(\gamma)=\int_{0}^{\gamma} \hat{\sigma}(\varepsilon) d \varepsilon, \quad \gamma>-1
$$


The following work-energy relation can be readily established:

$$
\sigma\left(x_{2}, t\right) v\left(x_{2}, t\right)-\sigma\left(x_{1}, t\right) v\left(x_{1}, t\right)-\dot{E}(t)=f(t) \dot{s}(t),
$$

where the driving force (or driving traction) $f(t)$ acting on the strain discontinuity is defined by

$$
f=\hat{f}(\bar{\gamma}, \stackrel{+}{\gamma}) \equiv \int_{\bar{\gamma}}^{+} \hat{\sigma}(\gamma) d \gamma-\frac{1}{2}(\hat{\sigma}(\stackrel{+}{\gamma})+\hat{\sigma}(\bar{\gamma}))(\stackrel{+}{\gamma}-\bar{\gamma}) .
$$

Note that $f$ may be interpreted geometrically as the difference between the area under the stress-strain curve between $\gamma=\bar{\gamma}$ and $\gamma=\stackrel{+}{\gamma}$ and the area of an associated trapezoid having the same base. The right-hand side of (6) represents the instantaneous dissipation rate due to the moving discontinuity and the requirement that it be nonnegative implies that

$$
f(t) \dot{s}(t) \geq 0 \text {. }
$$

Under isothermal conditions, the inequality $(8)$ is a consequence of the second law of thermodynamics.

A motion of the bar is governed by the field equations (2) at all points of smoothness, the jump conditions (3), and the entropy inequality (8) at all discontinuities.

3. Material. Shock waves and phase boundaries. In this paper, we consider a material whose stress response function $\hat{\sigma}(\gamma)$ is twice continuously differentiable with $\hat{\sigma}$ first increasing with increasing $\gamma$, then decreasing, and finally increasing again as shown in Fig. 1. More specifically, we suppose that there are three numbers $\gamma_{M}, \gamma_{m}$, and $\gamma_{i n}$ with $0<\gamma_{M}<\gamma_{i n}<\gamma_{m}$ such that

and

$$
\hat{\sigma}^{\prime}(\gamma) \begin{cases}>0, & -1<\gamma<\gamma_{M}, \\ =0, & \gamma=\gamma_{M}, \\ <0, & \gamma_{M}<\gamma<\gamma_{m}, \\ =0, & \gamma=\gamma_{m}, \\ >0, & \gamma>\gamma_{m},\end{cases}
$$

Moreover, we suppose that $\hat{\sigma}(0)=0$ and that

$$
\hat{\sigma}^{\prime \prime}(\gamma) \begin{cases}<0, & -1<\gamma<\gamma_{i n}, \\ =0, & \gamma=\gamma_{i n}, \\ >0, & \gamma>\gamma_{i n} .\end{cases}
$$

$$
\begin{array}{cl}
\hat{\sigma}(\gamma) \rightarrow-\infty, & \hat{\sigma}^{\prime}(\gamma) \rightarrow \infty \quad \text { as } \gamma \rightarrow-1 ; \\
\hat{\sigma}(\gamma)=\mu_{\infty} \gamma+\sigma_{T}+o(1) & \text { as } \gamma \rightarrow \infty,
\end{array}
$$

where $\mu_{\infty}(>0)$ and $\sigma_{T}$ are constants. The stress-strain curve therefore consists of three branches, two of which are rising, while the other is declining; it has a single inflection point at the strain-level $\gamma=\gamma_{\text {in }}$ and is asymptotic, at large tensile strains, to the straight line $\sigma=\mu_{\infty} \gamma+\sigma_{T}$. It is useful for later purposes to note that there are two unique values of strain, $R_{\infty}$ and $P_{\infty}$, such that

$$
\mu_{\infty} R_{\infty}+\sigma_{T}=\hat{\sigma}\left(R_{\infty}\right), \quad \hat{\sigma}^{\prime}\left(P_{\infty}\right)=\mu_{\infty}, \quad \text { where }-1<R_{\infty}<P_{\infty}<\gamma_{M} ;
$$




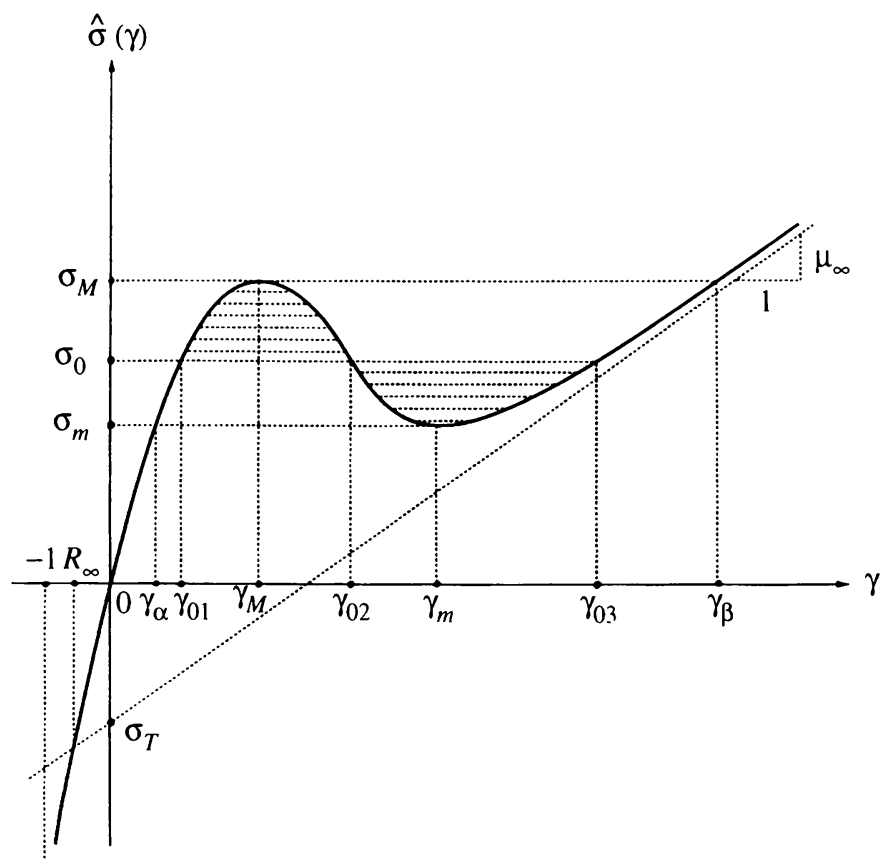

FIG. 1. Stress-strain curve

$R_{\infty}$ is the strain-level at which the asymptote $\sigma=\mu_{\infty} \gamma+\sigma_{T}$ intersects the first branch of the stress-strain curve, while $P_{\infty}$ is the value of strain on the first branch at which the slope equals the slope of this asymptote. Certain other material parameters are defined in the figure. In particular, the Maxwell stress $\sigma_{0}$ is the stress-level for which the two hatched areas of Fig. 1 are equal.

We shall say that a particle of the bar labeled by $x$ in the reference state is in the low-strain phase, the "unstable phase", or the high-strain phase at time $t$ during a motion if $\gamma(x, t)$ lies in the respective intervals $\left(-1, \gamma_{M}\right],\left(\gamma_{M}, \gamma_{m}\right)$, or $\left[\gamma_{m}, \infty\right)$. At a moving discontinuity $x=s(t)$, the jump conditions (3) imply

$$
\rho \dot{s}^{2}=\frac{\hat{\sigma}(\stackrel{+}{\gamma})-\hat{\sigma}(\bar{\gamma})}{\dot{+}-\bar{\gamma}} \quad(\geq 0) .
$$

A discontinuity is called either a shock wave or a phase boundary according to whether $\bar{\gamma}$ and $\stackrel{+}{\gamma}$ both lie in the same phase or in distinct phases. The sound speed of the material at a strain $\gamma$ is defined by

$$
c(\gamma)=\sqrt{\frac{\hat{\sigma}^{\prime}(\gamma)}{\rho}},
$$

where it is necessary that $\gamma$ in (15) not belong to the unstable phase. Let $c_{\infty}=$ $\sqrt{\mu_{\infty} / \rho}$. Let $\stackrel{ \pm}{c}=c\left(\frac{ \pm}{\gamma}\right)$ stand for the sound speeds on the two sides of a discontinuity. The propagation speed $\dot{s}$ of the discontinuity is said to be subsonic if $|\dot{s}|<\bar{c}$ and $\stackrel{+}{c}$, intersonic if $\bar{c}<|\dot{s}|<\stackrel{+}{c}$ or $\stackrel{+}{c}<|\dot{s}|<\bar{c}$, and supersonic if $|\dot{s}|>\bar{c}$ and $\stackrel{+}{c}$. 
We shall speak of a low-strain shock wave and a high-strain shock wave according to whether the strains $\bar{\gamma}, \stackrel{+}{\gamma}$ both belong to the low-strain phase or to the high-strain phase. For the material (9)-(12) considered here, it can be readily seen that all shock waves are intersonic. Moreover, it follows from (7) and (9)-(12) that the entropy inequality (8) holds at a shock wave if and only if

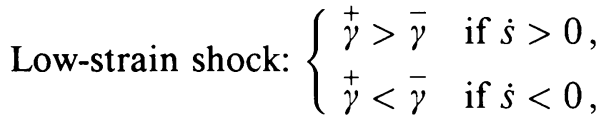

$$
\begin{aligned}
& \text { High-strain shock: } \begin{cases}\stackrel{+}{\gamma}<\bar{\gamma} & \text { if } \dot{s}>0, \\
\hat{\gamma}>\bar{\gamma} & \text { if } \dot{s}<0 .\end{cases}
\end{aligned}
$$

This implies, in particular, that a shock wave always moves into the phase whose sound speed is smaller than the speed $|\dot{s}|$ of the shock wave.

Turning next to phase boundaries, we will show in the next section that a phase boundary for which either $\bar{\gamma}$ or $\stackrel{+}{\gamma}$ is in the unstable phase cannot arise in the problem to be considered here. Thus, suppose that $\bar{\gamma}$ belongs to the high-strain phase and $\hat{\gamma}$ to the low-strain phase. In the $(\bar{\gamma}, \stackrel{+}{\gamma})$-plane, the set of all pairs $(\bar{\gamma}, \stackrel{+}{\gamma})$ for which $\bar{\gamma}$ is in the high-strain phase, $\stackrel{+}{\gamma}$ is in the low-strain phase and the right side of (14) is nonnegative is represented by the union $\Gamma$ of the hatched regions in Fig. 2; it is the region bounded by the lines $\stackrel{+}{\gamma}=-1, \stackrel{+}{\gamma}=\gamma_{M}, \bar{\gamma}=\gamma_{m}$ and the curve $\hat{\sigma}(\stackrel{+}{\gamma})=\hat{\sigma}(\bar{\gamma})$. The region $\Gamma$ of the $(\bar{\gamma}, \stackrel{+}{\gamma})$-plane will play a major role in the analysis that follows in the next sections. The boundary segment $\mathscr{E}$ is defined by

$$
\mathscr{E}: \hat{\sigma}(\stackrel{+}{\gamma})=\hat{\sigma}(\bar{\gamma}) \Leftrightarrow \stackrel{+}{\gamma}=T(\bar{\gamma}), \quad \gamma_{m} \leq \bar{\gamma} \leq \gamma_{\beta},
$$

where the material parameters $\gamma_{\beta}$ and $\gamma_{m}$ are defined in Fig. 1. By (14), $\dot{s}=0$ at points on $\mathscr{E}$ and so this segment represents instantaneously stationary or equilibrium states of the phase boundary. One can verify that $T^{\prime}(\gamma)>0$ for $\gamma_{m}<\gamma<\gamma_{\beta}$ and that $T^{\prime}\left(\gamma_{m}\right)=0, T^{\prime}\left(\gamma_{\beta}\right)=\infty$; the curve $\mathscr{E}$ therefore rises monotonically as $\bar{\gamma}$ increases. Next, consider the curve $\mathscr{F}$ which is defined as the set of points $(\bar{\gamma}, \stackrel{+}{\gamma})$ at which the driving force $\hat{f}(\bar{\gamma}, \stackrel{+}{\gamma})$ introduced in (7) vanishes:

$$
\mathscr{F}: \hat{f}(\bar{\gamma}, \stackrel{+}{\gamma})=0 \Leftrightarrow \stackrel{+}{\gamma}=Q(\bar{\gamma}), \quad \bar{\gamma} \geq \gamma_{03},
$$

where the material parameter $\gamma_{03}$ is defined in Fig. 1 . One can verify that $Q^{\prime}(\gamma)<0$ and that $Q(\gamma) \rightarrow R_{\infty}, Q^{\prime}(\gamma) \rightarrow 0$ as $\gamma \rightarrow \infty$ where $P_{\infty}$ is the value of strain defined previously in (13). The curve $\mathscr{F}$ therefore declines monotonically as $\bar{\gamma}$ increases as shown in the figure. In view of (8), a phase boundary associated with a point on $\mathscr{F}$ propagates without dissipation. Since $\hat{f}(\bar{\gamma}, \stackrel{+}{\gamma})>0$ above $\mathscr{F}$, the entropy inequality indicates that $\dot{s} \geq 0$ there; likewise $f<0$ and $\dot{s} \leq 0$ below $\mathscr{F}$. Consider next the curves $\stackrel{+}{\mathscr{S}}$ and $\overline{\mathscr{S}}$ that are defined as the ("sonic") curves on which the speed $\dot{s}$ of 


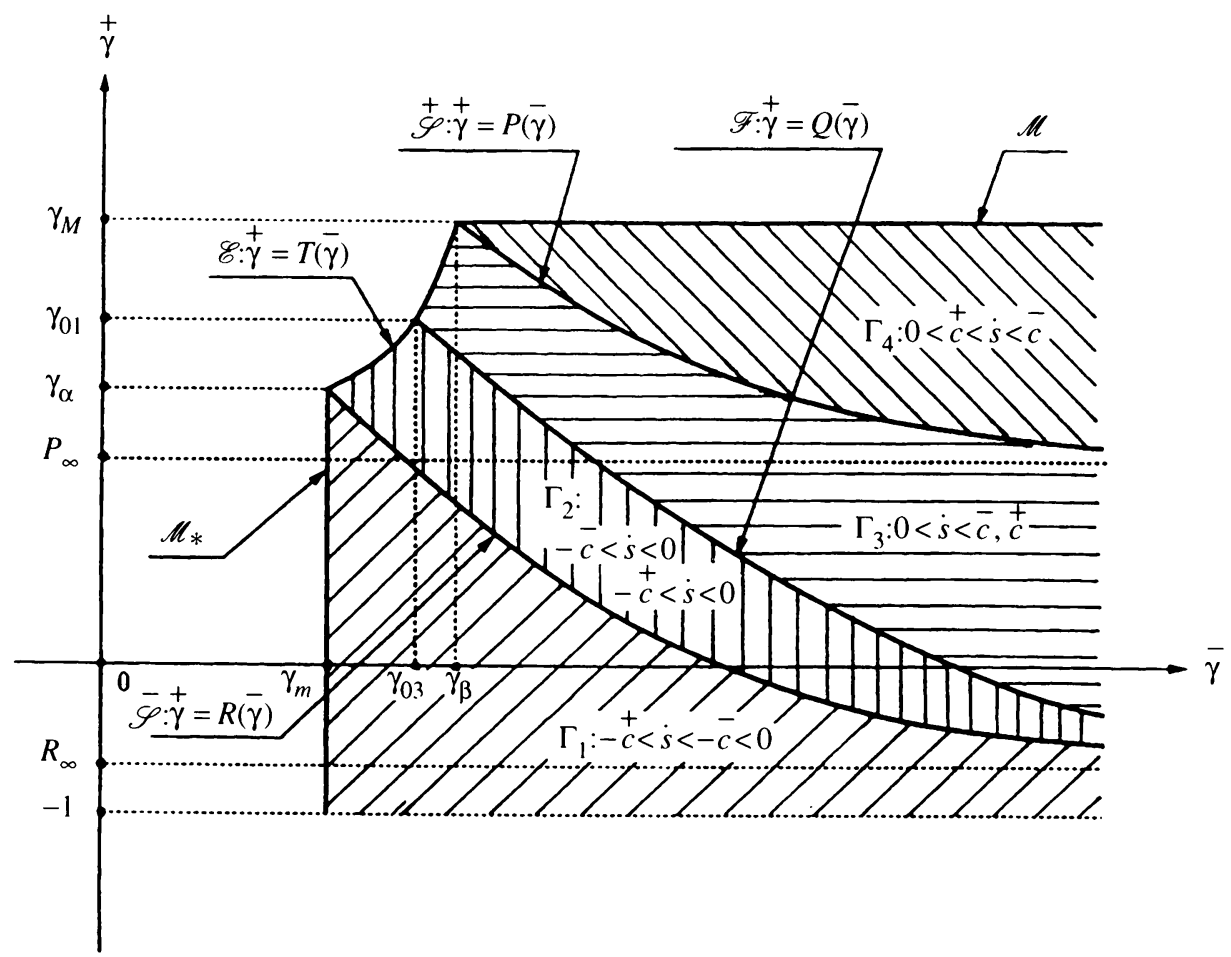

Fig. 2. The regions $\Gamma_{i}$ in the $(\bar{\gamma}, \stackrel{+}{\gamma})$-plane

the phase boundary is equal, respectively, to the sound speeds $\stackrel{+}{c}$ and $\bar{c}$ :

$$
\begin{aligned}
& \stackrel{+}{\mathscr{S}}: \sqrt{(\hat{\sigma}(\stackrel{+}{\gamma})-\hat{\sigma}(\bar{\gamma})) /(\grave{\gamma}-\bar{\gamma})}=\sqrt{\hat{\sigma}^{\prime}(\dot{+})} \Leftrightarrow \stackrel{+}{\gamma}=P(\bar{\gamma}), \quad \bar{\gamma} \geq \gamma_{\beta}, \\
& \overline{\mathscr{S}}: \sqrt{(\hat{\sigma}(\dot{\gamma})-\hat{\sigma}(\bar{\gamma})) /(\dot{\gamma}-\bar{\gamma})}=\sqrt{\hat{\sigma}^{\prime}(\bar{\gamma})} \Leftrightarrow \stackrel{+}{\gamma}=R(\bar{\gamma}), \quad \bar{\gamma} \geq \gamma_{m} .
\end{aligned}
$$

One can verify that $P^{\prime}(\gamma), R^{\prime}(\gamma)<0$ and that $P(\gamma) \rightarrow P_{\infty}, R(\gamma) \rightarrow R_{\infty}, P^{\prime}(\gamma), R^{\prime}(\gamma)$ $\rightarrow 0$ as $\gamma \rightarrow \infty$ where $P_{\infty}$ and $R_{\infty}$ were defined earlier in (13). The curves $\overline{\mathscr{S}}$ and $\stackrel{+}{\mathscr{S}}$ therefore decline monotonically with increasing $\bar{\gamma}$ as shown in Fig. 2. One can also verify that the three curves $\overline{\mathscr{S}}, \stackrel{+}{\mathscr{S}}$, and $\mathscr{F}$ do not intersect each other; necessarily, the curves $\mathscr{F}$ and $\overline{\mathscr{S}}$ approach each other asymptotically as $\bar{\gamma} \rightarrow \infty$. The region $\Gamma$ is thus divided into four subregions $\Gamma_{1}, \Gamma_{2}, \Gamma_{3}$, and $\Gamma_{4}$ by these curves. The regions $\Gamma_{1}$ and $\Gamma_{2}$ correspond to phase boundaries that propagate into the high-strain phase at, respectively, intersonic and subsonic speeds; the regions $\Gamma_{3}$ and $\Gamma_{4}$ correspond to phase boundaries that propagate into the low-strain phase at, respectively, subsonic and intersonic speeds. Points on the curves $\overline{\mathscr{S}}$ and $\dot{\mathscr{S}}$ correspond to sonic phase boundaries. For the material (9)-(12) under consideration, supersonic phase boundaries cannot occur. We note that the figure has been drawn for the case $\gamma_{\alpha}>P_{\infty}$ though we do not assume this in the analysis. 


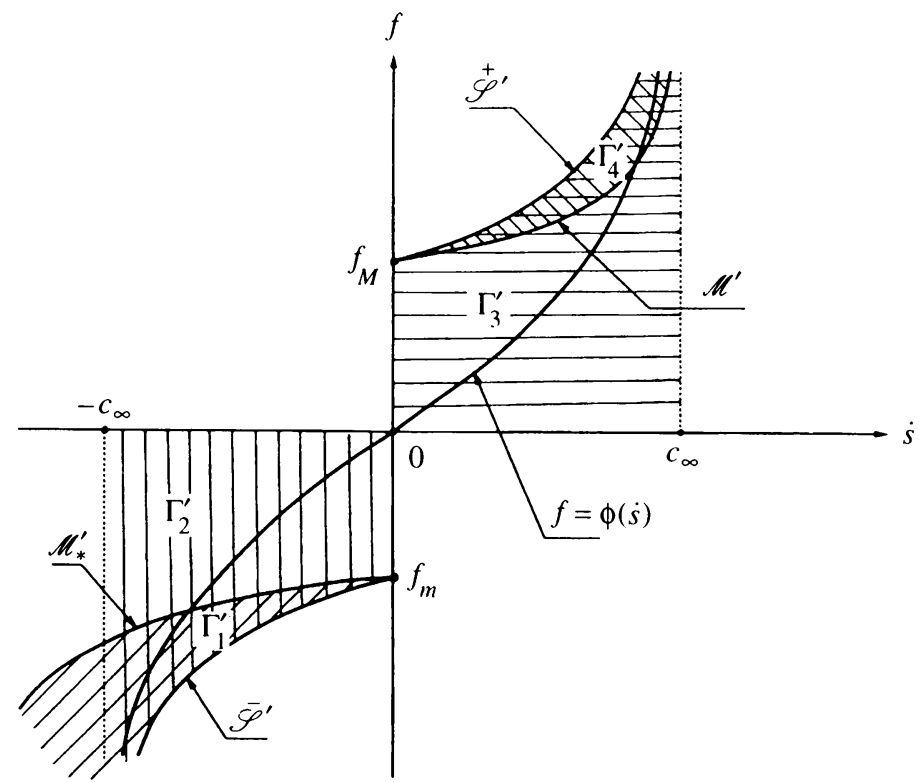

FIG. 3. Admissible images of $\Gamma_{i}$ in $(\dot{s}, f)$-plane

Finally, we consider the mapping $(\bar{\gamma}, \stackrel{+}{\gamma}) \rightarrow(\dot{s}, f)$ defined by (7), (8), and (14). One can verify that the Jacobian determinant of this mapping vanishes when $\bar{\gamma}, \dot{\gamma}$ corresponds to a sonic phase boundary, i.e., on the curves $\overline{\mathscr{S}}$ and $\stackrel{+}{\mathscr{S}}$. Considering the subsonic and intersonic regions separately, one can map each of the regions $\Gamma_{i}$ into the $(\dot{s}, f)$-plane; Fig. 3 shows the images $\Gamma_{i}^{\prime}$ that result from this mapping. Each of the curves $\overline{\mathscr{S}}^{\prime}, \stackrel{\mathscr{S}}{ }^{\prime}, \mathscr{M}^{\prime}$, and $\mathscr{M}_{*}^{\prime}$ rises monotonically as $\dot{s}$ increases. (The curves $\mathscr{M}^{\prime}$ and $\mathscr{M}_{*}^{\prime}$ are the images of the straight lines $\mathscr{M}$ and $\mathscr{M}_{*}$ shown in Fig. 2.) As $\dot{s} \rightarrow c_{\infty}$ the curves $\stackrel{\mathscr{S}}{ }^{\prime}$ and $\mathscr{M}^{\prime}$ rise without bound; when $\dot{s} \rightarrow-c_{\infty}$ the curve $\overline{\mathscr{S}}^{\prime}$ declines without bound. In Fig. $3, c_{\infty}=\sqrt{\mu_{\infty} / \rho}, f_{M}=\hat{f}\left(\gamma_{\beta}, \gamma_{M}\right)$, and $f_{m}=\hat{f}\left(\gamma_{m}, \gamma_{\alpha}\right)$. Note that though this mapping of $\Gamma$ from the $(\bar{\gamma}, \hat{\gamma})$-plane to the $(\dot{s}, f)$-plane is not one-to-one, when restricted to the subsonic region $\Gamma_{2} \cup \Gamma_{3}$, it is one-to-one.

4. The Riemann problem. Wave fans. We now formulate the Riemann problem for the field equations and jump conditions (2)-(3). We seek weak solutions of the differential equations $(2)$ on the upper half of the $(x, t)$-plane that satisfy the following initial conditions:

$$
\gamma(x, 0), v(x, 0)= \begin{cases}\gamma_{L}, v_{L}, & -\infty<x<0, \\ \gamma_{R}, v_{R}, & 0<x<\infty,\end{cases}
$$

where $\gamma_{L}, \gamma_{R}, v_{L}$, and $v_{R}$ are given constants with $\gamma_{L}>-1$ and $\gamma_{R}>-1$.

Since the initial value problem described above is invariant under the scale change $t \rightarrow k t, x \rightarrow k x$, we restrict attention to solutions that have this property as well. 
Where such solutions exist, they must have the form $\gamma(x, t)=\hat{\gamma}(\xi), v(x, t)=\hat{v}(\xi)$ where $\xi=x / t$. It then follows from (2) that for such solutions, either $\gamma$ and $v$ are both constant, or they are the wave fans that are given by

$$
\begin{gathered}
\hat{\sigma}^{\prime}(\hat{\gamma}(\xi))=\rho \xi^{2}, \\
\hat{v}^{\prime}(\xi)=-\xi \hat{\gamma}^{\prime}(\xi) .
\end{gathered}
$$

The left side of (22) must necessarily be nonnegative for any $\hat{\gamma}$ that satisfies (22). Thus, for the material (9)-(12), wave fans can occur only if $\hat{\gamma}$ takes values in either the low-strain phase or the high-strain phase. We shall speak of a low-strain fan or a high-strain fan according to whether $\hat{\gamma}$ belongs to the low-strain phase or the high-strain phase, respectively. In view of (9)-(12), it follows that (22) defines a unique function $\hat{\gamma}(\xi)=\gamma^{(L)}(\xi)$ for $-\infty<\xi<\infty$ such that $\gamma^{(L)} \in\left(-1, \gamma_{M}\right] ; \gamma^{(L)}$ describes the strain field in a low-strain fan. Similarly (22) can be uniquely solved for a function $\hat{\gamma}(\xi)=\gamma^{(H)}(\xi)$ for $-c_{\infty}<\xi<c_{\infty}$ such that $\gamma^{(H)} \in\left[\gamma_{m}, \infty\right) ; \gamma^{(H)}$ describes the strain field in a high-strain fan. Thus (22), (23) lead to the two wave fans

$$
\hat{\gamma}(\xi)=\gamma^{(i)}(\xi), \quad \hat{v}(\xi)=v^{(i)}(\xi), \quad i=L, H,
$$

where

$$
-\left(v^{(i)}(\xi)-v^{(i)}\left(\xi_{0}\right)\right)= \pm \int_{\gamma^{(i)}\left(\xi_{0}\right)}^{\gamma^{(i)}(\xi)} c(\gamma) d \gamma, \quad i=L, H,
$$

and $\xi_{0}$ describes an arbitrary ray $x / t=\xi_{0}$ within the fan; necessarily $\xi_{0}$ must lie in the interval $(-\infty, \infty)$ for a low-strain fan and in the interval $\left(-c_{\infty}, c_{\infty}\right)$ for a high-strain fan. The positive and negative signs are taken in the right side of (25) according to whether the wave fan occurs in the first quadrant or second quadrant of the $(x, t)$-plane, respectively.

Consider two rays $x=\bar{c} t$ and $x=\stackrel{+}{c} t$ with $\bar{c}<\stackrel{+}{c}$ in the $(x, t)$-plane between which the field is a fan. Let $\bar{\gamma}=\gamma(\bar{c} t+, t), \bar{v}=v(\bar{c} t+, t), \stackrel{+}{\gamma}=\gamma(\stackrel{+}{c} t-, t), \stackrel{+}{v}=$ $v(\stackrel{+}{c} t-, t)$ denote the limiting values from within the fan of strain and particle velocity at these rays. It follows from (15) and (22) that

$$
\bar{c}= \pm c(\bar{\gamma}), \quad \stackrel{+}{c}= \pm c(\stackrel{+}{\gamma}),
$$

and from (23) that

$$
-(\stackrel{+}{v}-\bar{v})= \pm \int_{\bar{\gamma}}^{+} c(\gamma) d \gamma,
$$

where the positive and negative signs are taken according to whether the fan occurs in the first or second quadrant of the $(x, t)$-plane, respectively. Equation (27) is the analog for a fan of the kinematic jump condition for a discontinuity in (3). Since the field within the fan is smooth, the entropy inequality is trivially satisfied at points 


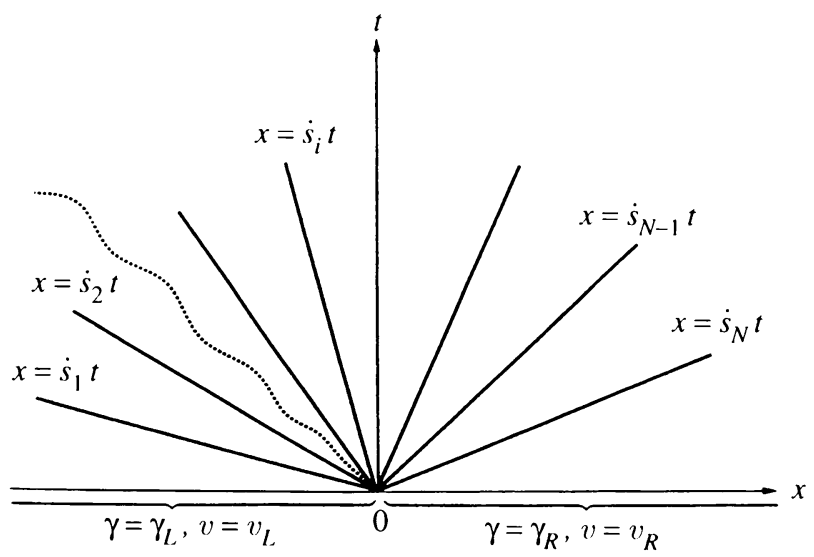

FIG. 4. General form of solutions to the Riemann problem

within it. For the material (9)-(12), it follows from (22) that necessarily

$$
\begin{gathered}
\text { Low-strain fan: }\left\{\begin{array}{l}
\begin{array}{l}
+ \\
\gamma
\end{array} \bar{\gamma} \text { if fan is in first quadrant, } \\
+\gamma>\bar{\gamma} \text { if fan is in second quadrant },
\end{array}\right. \\
\text { High-strain fan: }\left\{\begin{array}{l}
+>\bar{\gamma} \text { if fan is in first quadrant, } \\
+<\bar{\gamma} \text { if fan is in second quadrant. }
\end{array}\right.
\end{gathered}
$$

Equations (28)-(29) are the analog for fans of Eqs. (16)-(17) for shocks. Conversely, given numbers $(\bar{\gamma}, \bar{v}),(\stackrel{+}{\gamma}, \stackrel{+}{v})$ that conform to (27)-(29), one can construct a unique fan between the rays $x=\bar{c} t$ and $x=\stackrel{+}{c} t$ where $\stackrel{t}{c}$ is given by (26).

The general scale-invariant solution to the Riemann problem has the form shown in Fig. 4: between any two rays $x=\dot{s}_{i} t$ and $x=\dot{s}_{i+1} t$ the fields $\gamma, v$ are either constants or fans; the rays themselves may or may not correspond to discontinuities. If $x=\dot{s}_{i} t$ is a discontinuity, the jump conditions (3) must be satisfied across it so that

$$
\left\{\begin{array}{l}
\dot{s}_{i}\left(\gamma_{i}-\gamma_{i-1}\right)=-\left(v_{i}-v_{i-1}\right), \\
\rho \dot{s}_{i}^{2}=\left(\hat{\sigma}\left(\gamma_{i}\right)-\hat{\sigma}\left(\gamma_{i-1}\right)\right) /\left(\gamma_{i}-\gamma_{i-1}\right),
\end{array}\right.
$$

where $\left(\gamma_{i}, v_{i}\right)$ and $\left(\gamma_{i-1}, v_{i-1}\right)$ are the limiting values of strain and particle velocity on the right and left, respectively, of this discontinuity. Let $f_{i}=\hat{f}\left(\gamma_{i-1}, \gamma_{i}\right)$ with $\hat{f}$ defined by (7) standing for the driving force on this discontinuity; the entropy inequality $(8)$ then requires that

$$
f_{i} \dot{s}_{i} \geq 0 \text {. }
$$

An admissible solution of the Riemann problem is a pair $\gamma(x, t), v(x, t)$ of the form just described with (30)-(31) enforced at all discontinuities.

5. The structure of admissible solutions to the Riemann problem. We shall say that the initial data in $(21)$ is metastable if neither of the initial strains $\gamma_{L}, \gamma_{R}$ belong to the unstable phase. Before constructing explicit global solutions to the Riemann 
problem, it is helpful to establish some general results pertaining to the permissible solution forms that are consistent with the entropy inequality.

Let $(\gamma, v)$ be an admissible solution of the Riemann problem with metastable initial data.

(i) The strain $\gamma(x, t)$ does not belong to the unstable phase at any point $(x, t)$ in the upper-half plane.

This result implies that if the initial data does not involve the unstable phase, then at no later time does the solution involve the unstable phase. We prove this claim by contradiction. Suppose that this proposition is false. Since unstable phase fans and shocks do not exist, there must necessarily be two phase boundaries in the $(x, t)$ plane, say $x=\dot{s}_{k} t$ and $x=\dot{s}_{k+1} t$ with $\dot{s}_{k}<\dot{s}_{k+1}$, such that the state between them is constant with the associated strain $\gamma_{k}$ in the unstable phase and with neither of the strains $\gamma_{k-1}=\gamma\left(\dot{s}_{k} t-, t\right)$ and $\gamma_{k+1}=\gamma\left(\dot{s}_{k+1} t+, t\right)$ in the unstable phase. From (7) and (9)-(12) one sees that the driving force on the discontinuity $x=\dot{s}_{k} t$ is positive; the entropy inequality (31) thus implies that $\dot{s}_{k} \geq 0$. Similarly, the driving force on $x=\dot{s}_{k+1} t$ must be negative and so $\dot{s}_{k+1} \leq 0$. Thus $\dot{s}_{k} \geq \dot{s}_{k+1}$, which is a contradiction. This establishes the proposition.

The next six propositions are concerned with the possibility of having shock waves, phase boundaries, and wave fans adjacent to each other. When addressing fans, it is sufficient for our purposes to restrict attention to fans that do not terminate on discontinuities. Thus in the propositions that follow, if $x=\dot{s}_{k} t$ and $x=\dot{s}_{k+1} t$ are two rays in the $(x, t)$-plane between which the field is a fan, these two rays will be assumed not to be discontinuities. Features of fans that do terminate at discontinuities can be deduced from the following results by suitable limiting arguments.

Let $(\gamma, v)$ be an admissible solution of the Riemann problem with metastable initial data.

(ii) Let $x=\dot{s}_{k} t, x=\dot{s}_{k+1} t, x=\dot{s}_{k+2} t$, and $x=\dot{s}_{k+3} t$ be four rays in the same quadrant of the $(x, t)$-plane. If the field in the interior wedge $\dot{s}_{k+1} t<x<\dot{s}_{k+2} t$ is constant, then the fields in the other two wedges cannot both be fans.

(iii) Let $x=\dot{s}_{k} t$ and $x=\dot{s}_{k+1} t$ be two rays in the same quadrant of the $(x, t)$-plane between which the field is constant. Then these two rays cannot both be shock waves.

(iv) Let $x=\dot{s}_{k} t$ and $x=\dot{s}_{k+1} t$ be two rays in the same quadrant of the $(x, t)$-plane between which the field is constant. Then these two rays cannot both be phase boundaries.

(v) Let $x=\dot{s}_{k} t, x=\dot{s}_{k+1} t$, and $x=\dot{s}_{k+2} t$ be three rays in the same quadrant of the $(x, t)$-plane. Suppose that the field between any two of these rays is a fan. Then the third ray cannot be a shock. 
(vi) Let $x=\dot{s}_{k} t, x=\dot{s}_{k+1} t$, and $x=\dot{s}_{k+2} t$ be three rays in the same quadrant of the $(x, t)$-plane. Suppose that the field between the two slowest rays is a fan. Then the remaining ray cannot be a phase boundary. (The converse case is possible: if the field between the two fastest rays is a fan, the remaining ray may be a phase boundary.)

(vii) Let $x=\dot{s}_{k} t$ and $x=\dot{s}_{k+1} t$ be two rays in the same quadrant of the $(x, t)$-plane. If the slower ray is a shock, then the faster ray cannot be a phase boundary. (The converse case is possible: if the faster ray is a shock, the slower ray may be a phase boundary.)

The first three of these propositions state that two wave fans, two shock waves, and two phase boundaries cannot be adjacent to each other. The next one states that a fan and a shock cannot be adjacent to each other. On the other hand, according to Proposition (vi), a fan and a phase boundary may be adjacent to each other provided the phase boundary is subsonic. Similarly, a shock and a phase boundary may be adjacent to each other provided the phase boundary travels more slowly than the shock.

It is clearly sufficient to prove these results in any one quadrant of the upper half of the $(x, t)$-plane and so we shall consider only the first quadrant. Thus, in each of these propositions we have $0<\dot{s}_{k}<\dot{s}_{k+1}<\dot{s}_{k+2}<\dot{s}_{k+3}$.

To prove Proposition (ii), suppose that it is false so that the fields in $\dot{s}_{k} t<x<$ $\dot{s}_{k+1} t$ and $\dot{s}_{k+2} t<x<\dot{s}_{k+3} t$ are fans while the field is constant in the intermediate wedge. It is not possible that one of these fans is a low-strain fan while the other is a high-strain fan, since such fans belong to different phases and so must necessarily be separated by a phase boundary. For two fans of the same type, (22) directly shows that they must in fact be smoothly connected to each other to become a single fan with $\dot{s}_{k+1}=\dot{s}_{k+2}$. This contradicts the assumption that $\dot{s}_{k+1}<\dot{s}_{k+2}$. The assertion (ii) is thus proved.

We now turn to the proof of Proposition (iii). Suppose that the proposition is false so that $x=\dot{s}_{k} t$ and $x=\dot{s}_{k+1} t$ are both shocks and the field between them is constant. Note first that a low-strain shock cannot be adjacent to a high-strain shock, since they must be separated by a phase boundary. Suppose that both $x=\dot{s}_{k} t$ and $x=\dot{s}_{k+1} t$ are low-strain shocks. The strain and velocity fields thus have the form

$$
\gamma(x, t), v(x, t)= \begin{cases}\gamma_{k-1}, v_{k-1} & x=\dot{s}_{k} t-, \\ \gamma_{k}, v_{k}, & \dot{s}_{k} t<x<\dot{s}_{k+1} t, \\ \gamma_{k+1}, v_{k+1}, & x=\dot{s}_{k+1} t+,\end{cases}
$$

where $\gamma_{k-1}, \gamma_{k}$, and $\gamma_{k+1}$ are distinct and all three belong to $\left(-1, \gamma_{M}\right]$. According to (31), the driving forces acting on these two shock waves must be nonnegative. With the help of (7) one finds that this implies that $\gamma_{k-1}<\gamma_{k}<\gamma_{k+1}$. Since $\hat{\sigma}^{\prime}(\gamma)>0$ and $\hat{\sigma}^{\prime \prime}(\gamma)<0$ on $\left(-1, \gamma_{M}\right)$, this, together with the fact that $-1<\gamma_{k-1}<\gamma_{k}<$ $\gamma_{k+1} \leq \gamma_{M}$, implies that

$$
\frac{\hat{\boldsymbol{\sigma}}\left(\gamma_{k+1}\right)-\hat{\boldsymbol{\sigma}}\left(\gamma_{k}\right)}{\gamma_{k+1}-\gamma_{k}}<\frac{\hat{\boldsymbol{\sigma}}\left(\gamma_{k}\right)-\hat{\sigma}\left(\gamma_{k-1}\right)}{\gamma_{k}-\gamma_{k-1}} .
$$


Equations (30) and (33) then yield $\dot{s}_{k}>\dot{s}_{k+1}$, which is a contradiction. In a similar way, we can prove that two high-strain shocks cannot be adjacent to each other. This proves Proposition (iii).

Propositions (iv) and (vii) may be established by arguments that are very similar to the above.

Next we turn to the proof of Proposition (v). Suppose that it is false. Note first that a low-strain shock cannot be adjacent to a high-strain fan (or vice versa) since they must be separated by a phase boundary. Suppose that $x=\dot{s}_{k} t$ is a low-strain shock and that the field between the rays $x=\dot{s}_{k+1} t$ and $x=\dot{s}_{k+2} t$ is a low-strain fan:

$$
\gamma(x, t), v(x, t)= \begin{cases}\gamma_{k-1}, v_{k-1}, & x=\dot{s}_{k} t-, \\ \gamma_{k}, v_{k}, & \dot{s}_{k} t<x \leq \dot{s}_{k+1} t, \\ \gamma^{(L)}(x / t), v^{(L)}(x / t), & \dot{s}_{k+1} t \leq x \leq \dot{s}_{k+2} t \\ \gamma_{k+2}, v_{k+2}, & x=\dot{s}_{k+2} t+,\end{cases}
$$

where $\gamma_{k-1}, \gamma_{k}$, and $\gamma_{k+2}$ all lie in the low-strain phase and $\gamma^{(L)}$ is the strain field in a low-strain fan given by (24). For the material (9)-(12), Eq. (22) and the fact that $\dot{s}_{k+1}<\dot{s}_{k+2}$ implies that $\gamma_{k}>\gamma_{k+2}$. Next, the entropy inequality (31) requires the driving force $\tilde{f}\left(\gamma_{k-1}, \gamma_{k}\right)$ at $x=\dot{s}_{k} t$ to be nonnegative; by (7), this implies that $\gamma_{k-1}<\gamma_{k}$. Since $\hat{\sigma}^{\prime}(\gamma)>0$ and $\hat{\sigma}^{\prime \prime}(\gamma)<0$ on $\left[\gamma_{k-1}, \gamma_{k}\right]$, this, together with the fact that $-1<\gamma_{k-1}<\gamma_{k} \leq \gamma_{M}$ implies that

$$
\hat{\sigma}^{\prime}\left(\gamma_{k}\right)<\frac{\hat{\sigma}\left(\gamma_{k}\right)-\hat{\sigma}\left(\gamma_{k-1}\right)}{\gamma_{k}-\gamma_{k-1}} .
$$

Equations (15), (30) now yield $\dot{s}_{k}>\dot{s}_{k+1}$, which is a contradiction.

The remaining cases where $x=\dot{s}_{k+2} t$ is a low-strain shock and the field between the rays $x=\dot{s}_{k} t$ and $x=\dot{s}_{k+1} t$ is a low-strain fan, and when the shock and fan are both high-strain ones, can be treated similarly. This establishes Proposition (v).

The proof of Proposition (vi) is entirely analogous.

The preceding results imply that the form of admissible solutions to the Riemann problem with metastable initial data is in fact much simpler than that described in Fig. 4. Note that the results (iii)-(vii) depend critically on the entropy inequality (31).

6. Explicit solutions to the Riemann problem. The results established in the preceding section allow one to determine all admissible solutions to the Riemann problem in the case of metastable initial data. From here on we shall consider only the special Riemann problem in which the initial strains $\gamma_{L}$ and $\gamma_{R}$ are both in the low-strain phase, and $\gamma_{R}$ is smaller than $\gamma_{L}$ :

$$
\gamma_{L} \in\left(-1, \gamma_{M}\right], \quad \gamma_{R} \in\left(-1, \gamma_{M}\right], \quad \gamma_{R}<\gamma_{L}
$$




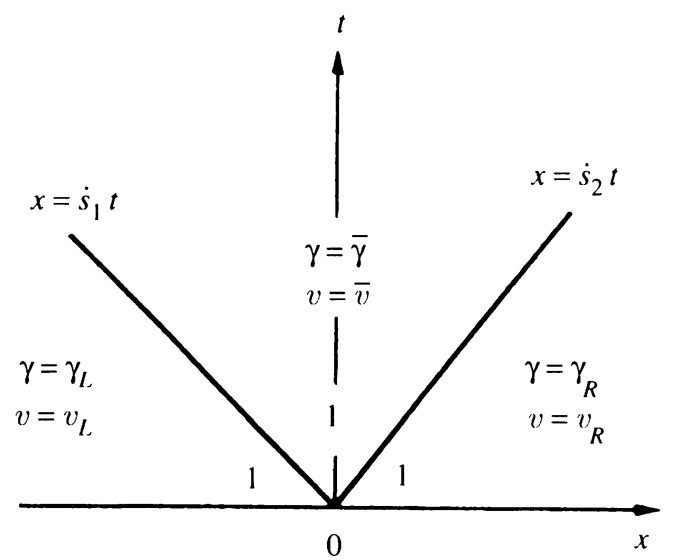

(a) Two shock waves

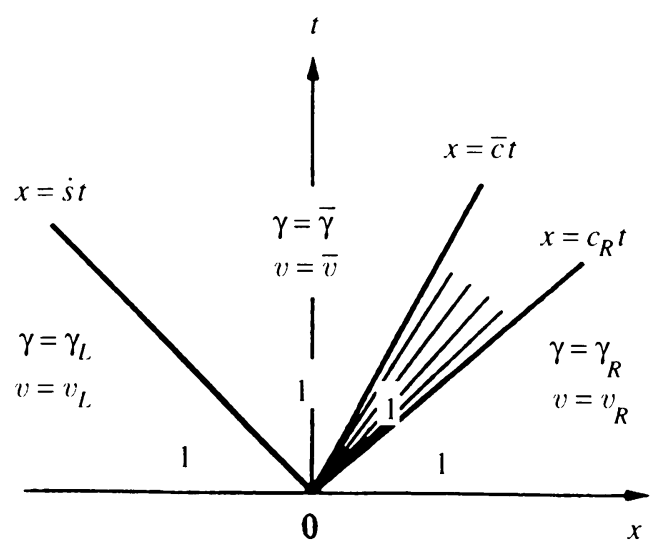

(b) One shock wave and one wave fan

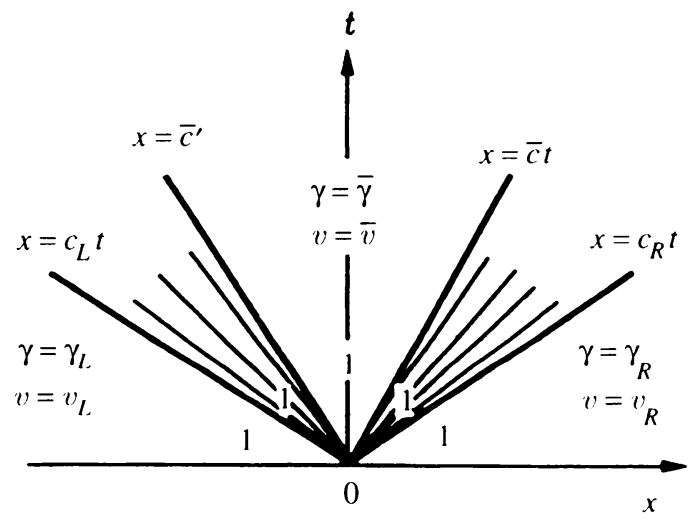

(c) Two wave fans

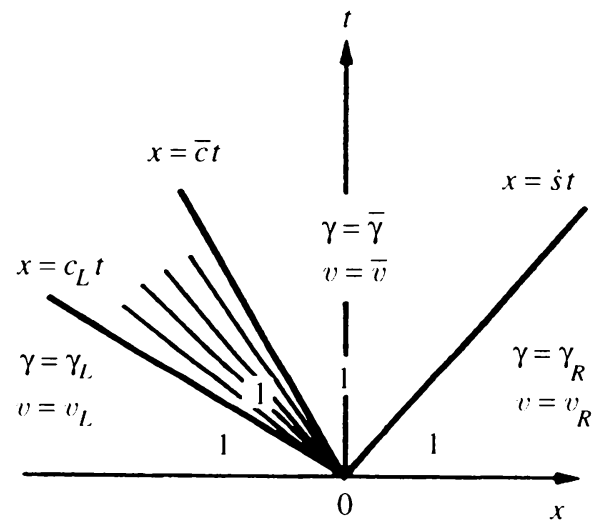

(d) One wave fan and one shock wave

Fig. 5. Form of solutions to Riemann problem without phase change. 1 = low-strain phase; 3 = high-strain phase

At the initial instant, the entire bar is in the low-strain phase. At a later instant, a particle of the bar may or may not change its phase. It is convenient in the following analysis to consider these two cases separately.

(i) Solutions involving no phase change. In this case, the solution does not involve any phase boundaries. In view of the first proposition in Sec. 5, neither does it involve the unstable phase at any time $t>0$. Next, in view of Propositions (ii), (iii), and (v), the solution $\gamma, v$ can only involve a single low-strain shock wave or a single lowstrain fan in each quadrant of the upper half of the $(x, t)$-plane. Thus the solution must have one of the four forms shown in Fig. 5(a)-(d). 
(i(a)) Solution with two shock waves. Figure 5(a). Consider a solution having the form shown in Fig. 5(a):

$$
\gamma, v= \begin{cases}\gamma_{L}, v_{L}, & -\infty<x<\dot{s}_{1} t, \\ \bar{\gamma}, \bar{v}, & \dot{s}_{1} t<x<\dot{s}_{2} t, \\ \gamma_{R}, v_{R}, & \dot{s}_{2} t<x<\infty,\end{cases}
$$

in which $\bar{\gamma}, \bar{v}, \dot{s}_{1}$, and $\dot{s}_{2}$ are to be found such that $\bar{\gamma} \in\left(-1, \gamma_{M}\right.$ ] and $\dot{s}_{1}<0<\dot{s}_{2}$.

The jump conditions (30) and the entropy inequality (31) at each of the two shock waves require that

$$
\begin{aligned}
& -\left(v_{R}-\bar{v}\right)=\dot{s}_{2}\left(\gamma_{R}-\bar{\gamma}\right), \quad \dot{s}_{2}=\sqrt{\frac{\hat{\sigma}\left(\gamma_{R}\right)-\hat{\sigma}(\bar{\gamma})}{\rho\left(\gamma_{R}-\bar{\gamma}\right)},} \quad \gamma_{R}>\bar{\gamma}, \\
& -\left(\bar{v}-v_{L}\right)=\dot{s}_{1}\left(\bar{\gamma}-\gamma_{L}\right), \quad \dot{s}_{1}=-\sqrt{\frac{\hat{\sigma}(\bar{\gamma})-\hat{\sigma}\left(\gamma_{L}\right)}{\rho\left(\bar{\gamma}-\gamma_{L}\right)}}, \quad \bar{\gamma}<\gamma_{L} .
\end{aligned}
$$

The inequalities in (36), (38), (39), together with the requirement that $\bar{\gamma}$ be in the low-strain phase, imply that

Combining (38)-(39) yields

$$
-1<\bar{\gamma}<\gamma_{R} .
$$

where $H(\gamma)$ is defined on $\left(-1, \gamma_{R}\right]$ by

$$
v_{R}-v_{L}=H(\bar{\gamma})
$$

$$
H(\gamma) \equiv-\sqrt{\left[\hat{\sigma}\left(\gamma_{R}\right)-\hat{\sigma}(\gamma)\right]\left(\gamma_{R}-\gamma\right) / \rho}-\sqrt{\left[\hat{\sigma}(\gamma)-\hat{\sigma}\left(\gamma_{L}\right)\right]\left(\gamma-\gamma_{L}\right) / \rho} .
$$

It can be verified that $H(\gamma)$ increases monotonically on $\left(-1, \gamma_{R}\right.$ ] from the value $-\infty$ at $\gamma=-1$ to the value $H_{R} \equiv H\left(\gamma_{R}\right)$ at $\gamma=\gamma_{R}$; see Lin [19]. Thus, if the initial data is such that $-\infty<v_{R}-v_{L}<H_{R}$, there is a unique root $\bar{\gamma}$ of (41) in the range $-1<\bar{\gamma}<\gamma_{R}\left(\leq \gamma_{M}\right)$. The remaining unknowns $\bar{v}, \dot{s}_{1}$, and $\dot{s}_{2}$ are then given immediately by (38) and (39).

Thus, there exists a unique admissible solution of the form (37) corresponding to Fig. 5 (a) if and only if the given initial data (21), (36) is such that $-\infty<v_{R}-v_{L}<H_{R}$.

(i(b)) Solution with a shock wave and a wave fan. Figure 5(b). Consider next a solution of the form shown in Fig. 5(b):

$$
\gamma, v= \begin{cases}\gamma_{L}, v_{L}, & -\infty<x<\dot{s} t, \\ \bar{\gamma}, \bar{v}, & \dot{s} t<x \leq \bar{c} t, \\ \hat{\gamma}(x / t), \hat{v}(x / t), & \bar{c} t \leq x \leq c_{R} t, \\ \gamma_{R}, v_{R}, & c_{R} t \leq x<\infty,\end{cases}
$$

where $\bar{\gamma}, \bar{v}, \dot{s}, \bar{c}$, and $c_{R}$ are to be determined such that $\bar{\gamma} \in\left(-1, \gamma_{M}\right]$ and $\dot{s}<$ $0<\bar{c}<c_{R}$. The functions $\hat{\gamma}(x / t)$ and $\hat{v}(x / t)$ are the strain and velocity fields pertaining to a low-strain fan and are given by $(24)-(25)$. 
At the shock wave $x=\dot{s} t$, the jump conditions (30) and the entropy inequality (31) must hold:

$$
\bar{v}=v_{L}-\dot{s}\left(\bar{\gamma}-\gamma_{L}\right), \quad \dot{s}=-\sqrt{\frac{\hat{\sigma}(\bar{\gamma})-\hat{\sigma}\left(\gamma_{L}\right)}{\rho\left(\bar{\gamma}-\gamma_{L}\right)}}, \quad \bar{\gamma}<\gamma_{L} .
$$

Turning next to the fan, and by using (26)-(28), one finds

$$
-\left(\bar{v}-v_{R}\right)=\int_{\gamma_{R}}^{\bar{\gamma}} c(\gamma) d \gamma, \quad c_{R}=c\left(\gamma_{R}\right), \quad \bar{c}=c(\bar{\gamma}), \quad \bar{\gamma}>\gamma_{R} .
$$

Equations (44)-(45) may now be combined to yield

$$
v_{R}-v_{L}=H(\bar{\gamma})
$$

where

$$
H(\gamma) \equiv-\sqrt{\left[\hat{\sigma}\left(\gamma_{L}\right)-\hat{\sigma}(\gamma)\right]\left(\gamma_{L}-\gamma\right) / \rho}+\int_{\gamma_{R}}^{\gamma} c(\varepsilon) d \varepsilon, \quad \gamma_{R} \leq \gamma \leq \gamma_{L} .
$$

One can verify that $H(\gamma)$ increases monotonically on $\left[\gamma_{R}, \gamma_{L}\right]$ from the value $H_{R} \equiv$ $H\left(\gamma_{R}\right)$ at $\gamma=\gamma_{R}$ to the value $H_{L} \equiv H\left(\gamma_{L}\right)$ at $\gamma=\gamma_{L}$; see [19]. Thus, if the initial data (21), (36) is such that $H_{R}<v_{R}-v_{L}<H_{L}$, Eq. (46) yields a unique root $\bar{\gamma}$ in the interval $\gamma_{R}<\bar{\gamma}<\gamma_{L}$. The remaining unknowns $\bar{v}, \dot{s}, \bar{c}$, and $c_{R}$ are then given by (44), (45).

Thus, there is a unique admissible solution of the form (43) corresponding to Fig. 5(b) if and only if the given initial data (21), (36) is such that $H_{R}<v_{R}-v_{L}<H_{L}$.

(i(c)) Solution with two wave fans. Figure 5(c). We seek solutions in the form of Fig. 5(c):

$$
\gamma, v= \begin{cases}\gamma_{L}, v_{L}, & -\infty<x \leq c_{L} t, \\ \hat{\gamma}_{1}(x / t), \hat{v}_{1}(x / t), & c_{L} t \leq x \leq \bar{c}^{\prime} t, \\ \bar{\gamma}, \bar{v}, & \bar{c}^{\prime} t \leq x \leq \bar{c} t \\ \hat{\gamma}_{2}(x / t), \hat{v}_{2}(x / t), & \bar{c} t \leq x \leq c_{R} t, \\ \gamma_{R}, v_{R}, & c_{R} t \leq x<\infty\end{cases}
$$

where $\bar{\gamma}, \bar{v}, c_{L}, \bar{c}^{\prime}, \bar{c}$, and $c_{R}$ are to be determined such that $\bar{\gamma} \in\left(-1, \gamma_{M}\right]$ and $c_{L}<\bar{c}^{\prime}<0<\bar{c}<c_{R}$. The functions $\hat{\gamma}_{1}, \hat{v}_{1}, \hat{\gamma}_{2}, \hat{v}_{2}$ are the fields that are appropriate to a low-strain fan and are given by (24)-(25).

The analysis of this case is similar to that of the preceding one. One finds that there is a unique admissible solution of the form (48) corresponding to Fig. 5(c) if and only if the given initial data (21), (36) is such that $H_{L}<v_{R}-v_{L}<H_{M}$ where

$$
H(\gamma) \equiv \int_{\gamma_{R}}^{\gamma} c(\gamma) d \gamma+\int_{\gamma_{L}}^{\gamma} c(\gamma) d \gamma, \quad \gamma_{L} \leq \gamma \leq \gamma_{M},
$$

and $H_{L} \equiv H\left(\gamma_{L}\right), H_{M} \equiv H\left(\gamma_{M}\right)$. 
(i(d)) Solution with a shock wave and a wave fan. Figure 5(d). For initial strains in the low-strain phase with $\gamma_{R}<\gamma_{L}$ as considered here, one finds that solutions having the form of Fig. 5(d) do not exist. In the reverse case $\gamma_{R}>\gamma_{L}$, one finds that such solutions do exist in place of solutions of the form in Fig. 5(b) which now do not exist.

It is readily seen from (42), (47), and (49) that $H(\gamma)$ is continuous at $\gamma=\gamma_{R}$ and $\gamma=\gamma_{L}$. In the respective limits $v_{R}-v_{L} \rightarrow H_{R}-$ and $v_{R}-v_{L} \rightarrow H_{R}+$, the solution forms shown in Fig. 5(a) and Fig. 5(b) coincide. The limiting solution involves a single shock wave traveling left and has no other shock waves. Similarly, the solution forms in Fig. 5(b) and Fig. 5(c) coincide in the respective limits $v_{R}-v_{L}=$ $H_{L^{-}}, v_{R}-v_{L}=H_{L^{+}}$. In this case the solution involves a single rightward moving wave fan.

In summary, when initial data (21), (36) is such that $-\infty<v_{R}-v_{L}<H_{M}$, it has been shown that there is a unique admissible solution that involves no phase change to the Riemann problem. Further discussion of these solutions is postponed until subsection (iii).

In order to find solutions when the initial data is such that $v_{R}-v_{L}>H_{M}$ we must consider solutions that involve a phase change.

(ii) Solutions involving a phase change. If a solution involves a phase change, the results (iv), (vi), and (vii) of Sec. 5 show that each quadrant of the upper half of the $(x, t)$-plane has precisely one phase boundary together with either one shock wave or one wave fan. Moreover, each phase boundary is necessarily subsonic so that the speed of the shock wave or wave fan is greater than that of the phase boundary. Thus the solution must have one of the four forms shown in Fig. 6(a)-(d). (See p. 592.)

(ii(a)) Solutions with two phase boundaries and two fans. Figure 6(a). Suppose that the solution has the form shown in Fig. 6(a):

$$
\gamma, v= \begin{cases}\gamma_{L}, v_{L}, & -\infty<x \leq c_{L} t, \\ \hat{\gamma}_{1}(x / t), \hat{v}_{1}(x / t), & c_{L} t \leq x \leq c_{A} t, \\ \gamma_{A}, v_{A}, & c_{A} t \leq x<\dot{s}_{1} t, \\ \bar{\gamma}, \bar{v}, & \dot{s}_{1} t<x<\dot{s}_{2} t, \\ \dot{\gamma}, \dot{v}, & \dot{s}_{2} t<x \leq \dot{+} t, \\ \hat{\gamma}_{2}(x / t), \hat{v}_{2}(x / t), & \stackrel{+}{c} t \leq x \leq c_{R} t, \\ \gamma_{R}, v_{R}, & c_{R} t \leq x<\infty,\end{cases}
$$

where $\gamma_{A}, v_{A}, \bar{\gamma}, \bar{v}, \stackrel{+}{\gamma}, \stackrel{+}{v}, c_{L}, c_{A}, \stackrel{+}{c}, c_{R}, \dot{s}_{1}$, and $\dot{s}_{2}$ are to be determined such that $\gamma_{A}, \stackrel{+}{\gamma} \in\left(-1, \gamma_{M}\right], \bar{\gamma} \in\left[\gamma_{m}, \infty\right)$, and $c_{L}<c_{A}<\dot{s}_{1} \leq 0 \leq \dot{s}_{2}<\stackrel{+}{c}<c_{R}$. The functions $\hat{\gamma}_{1}(x / t), \hat{v}_{1}(x / t), \hat{\gamma}_{2}(x / t), \hat{v}_{2}(x / t)$ are the fields appropriate to a low-strain fan and are given by (24)-(25). 


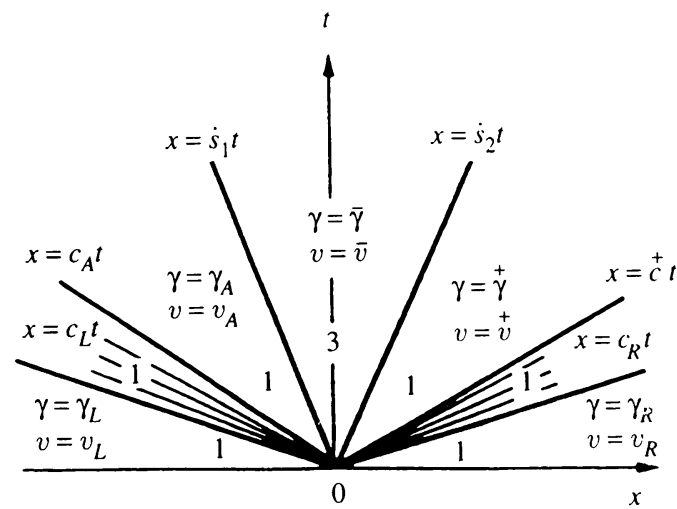

(a) Two phase boundaries and two wave fans

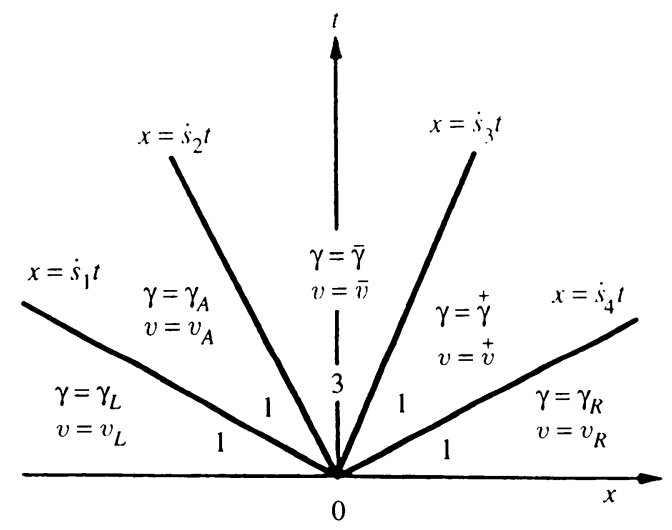

(c) Two phase boundaries and two shock waves

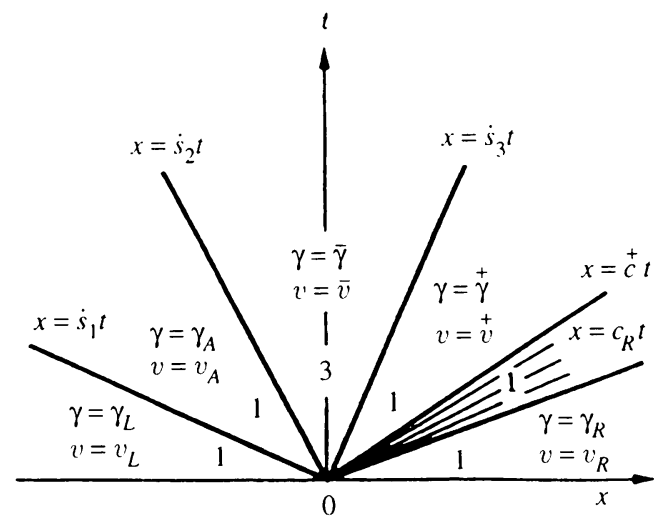

(b) Two phase boundaries, one shock wave, and one wave fan

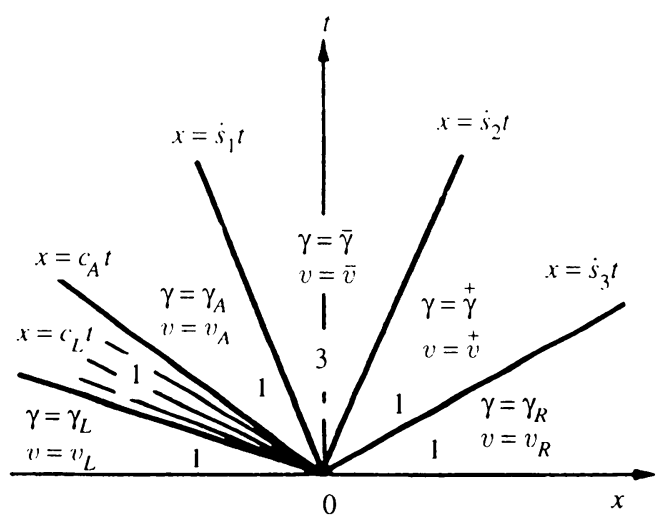

(d) Two phase boundaries, one wave fan, and one shock wave

FIG. 6. Form of solutions to Riemann problem with phase change.

$1=$ low-strain phase; 3 = high-strain phase

The jump conditions (30) at the two phase boundaries lead to

$$
\begin{array}{ll}
\stackrel{+}{v}=\bar{v}-\dot{s}_{2}(\dot{+}-\bar{\gamma}), & \dot{s}_{2}=\sqrt{\frac{\hat{\sigma}(\hat{\gamma})-\hat{\sigma}(\bar{\gamma})}{\rho(\dot{+}-\bar{\gamma})}}, \\
\bar{v}=v_{A}-\dot{s}_{1}\left(\bar{\gamma}-\gamma_{A}\right), & \dot{s}_{1}=-\sqrt{\frac{\hat{\sigma}(\bar{\gamma})-\hat{\sigma}\left(\gamma_{A}\right)}{\rho\left(\bar{\gamma}-\gamma_{A}\right)}},
\end{array}
$$


while the requirements $(26),(27)$ at the two fans give

$$
\begin{array}{lll}
\stackrel{+}{c}=c(\stackrel{+}{\gamma}), & c_{R}=c\left(\gamma_{R}\right), & -\left(\stackrel{+}{v}-v_{R}\right)=\int_{\gamma_{R}}^{+} c(\gamma) d \gamma, \\
c_{A}=-c\left(\gamma_{A}\right), & c_{L}=-c\left(\gamma_{L}\right), & v_{A}-v_{L}=\int_{\gamma_{L}}^{\gamma_{A}} c(\gamma) d \gamma .
\end{array}
$$

The roots of these equations are subject to the restrictions imposed by the entropy inequality (31) at each phase boundary, the inequality (28) at each fan, and the requirement that the strains belong to either low-strain phase or high-strain phase as appropriate. These restrictions lead to the following inequalities:

$$
\begin{gathered}
\hat{f}(\bar{\gamma}, \stackrel{+}{\gamma}) \geq 0, \quad \hat{f}\left(\gamma_{A}, \bar{\gamma}\right) \leq 0, \\
\stackrel{+}{\gamma}>\gamma_{R}, \quad \gamma_{A}>\gamma_{L}, \\
-1<\stackrel{+}{\gamma} \leq \gamma_{M}, \quad-1<\gamma_{a} \leq \gamma_{m}, \quad \bar{\gamma} \geq \gamma_{m} .
\end{gathered}
$$

The ten equations (51)-(54) are to be solved for the twelve unknown quantities listed above (50). Therefore, one anticipates that when there exists a solution, there would in fact be a two-parameter family of solutions; see James [12]. For reasons of algebraic simplicity, we assume that

$$
\dot{s}_{2}=-\dot{s}_{1} \equiv \dot{s},
$$

where $\dot{s} \geq 0$. This assumption does not change the essential characteristics of the solutions, but leads to a considerable simplification in the analysis. In fact, it is possible to show (as in Abeyaratne and Knowles [3]) that (58) necessarily holds once a kinetic relation is imposed. One now expects a one-, rather than a two-parameter family of solutions. In what follows, the strain $\bar{\gamma}$ will be treated as this parameter. The assumption (58) also leads to $\gamma_{A}=\stackrel{+}{\gamma}$.

It follows from (55)-(58) and the requirement $0 \leq \dot{s}<\stackrel{+}{c}$ in (50) that

$$
c(\stackrel{+}{\gamma})>\dot{s}, \quad \hat{f}(\bar{\gamma}, \stackrel{+}{\gamma}) \geq 0, \quad \stackrel{+}{\gamma}>\gamma_{L}, \quad \hat{\sigma}(\bar{\gamma})>\hat{\sigma}(\stackrel{+}{\gamma})
$$

necessarily hold. These inequalities define a region $D_{1} \subset \Gamma_{3}$ in the $(\bar{\gamma}, \stackrel{+}{\gamma})$-plane. Figure 7 displays this region in the case

$$
\gamma_{\alpha}>P_{\infty}>\gamma_{L}, \quad \gamma_{R}>R_{\infty},
$$

and for reasons of definiteness we shall frame our analysis from hereon for this particular case. The analysis can be trivially modified to handle the case when the ordering of the strains differs from (60).

By combining equations (51)-(54), one can reduce the question of their solvability to the following problem: given $\bar{\gamma}$, find a root $\stackrel{+}{\gamma}$ with $(\bar{\gamma}, \stackrel{+}{\gamma}) \in D_{1}$ of the equation

$$
v_{R}-v_{L}=G(\bar{\gamma}, \stackrel{+}{\gamma}),
$$




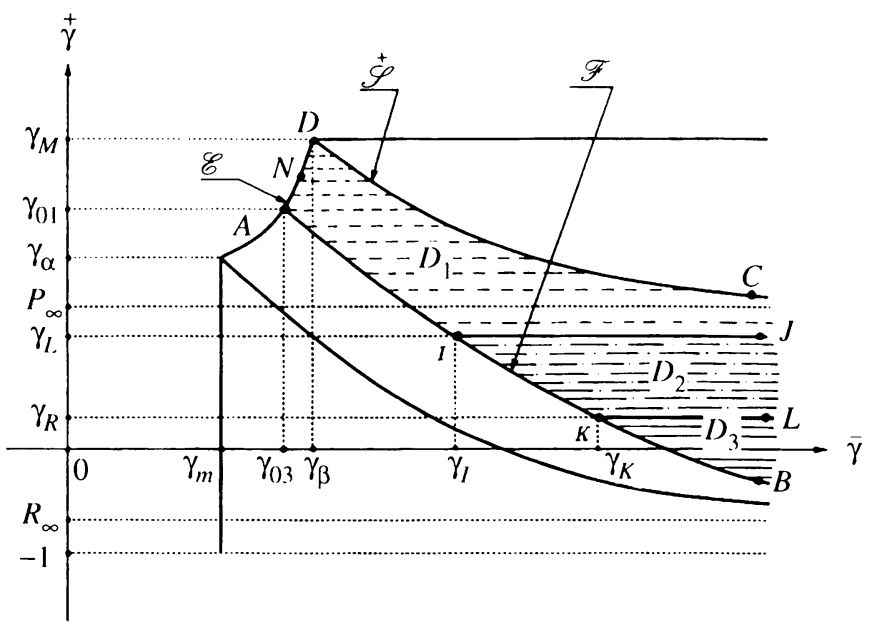

FIG. 7. The regions $D_{i}$ in the $(\bar{\gamma}, \stackrel{+}{\gamma})$-plane

where $G$ is defined for $(\bar{\gamma}, \stackrel{+}{\gamma}) \in D_{1}$ by

$$
G(\bar{\gamma}, \stackrel{+}{\gamma}) \equiv \int_{\gamma_{L}}^{+} c(\gamma) d \gamma+\int_{\gamma_{R}}^{+} c(\gamma) d \gamma+2 \sqrt{[\hat{\sigma}(\dot{\gamma})-\hat{\sigma}(\bar{\gamma})](\stackrel{+}{\gamma}-\bar{\gamma}) / \rho} .
$$

The curves $A D, A I$, and $C D$ of Fig. 7 which comprise part of the boundary of the region $D_{1}$ were defined previously in (18)-(20). At each fixed $\bar{\gamma}>\gamma_{03}$, the function $G$ in (62) increases monotonically with increasing $\dot{\gamma}$. Therefore, for each $\bar{\gamma} \geq \gamma_{03}$, (61) can be solved uniquely for ${ }^{+}$provided $v_{R}-v_{L}$ lies in a suitable range: for $\gamma_{03} \leq \bar{\gamma} \leq \gamma_{\beta}$, this range is $G(\bar{\gamma}, Q(\bar{\gamma})) \leq v_{R}-v_{L} \leq G(\bar{\gamma}, T(\bar{\gamma}))$; for $\gamma_{\beta} \leq \bar{\gamma}<\gamma_{I}$, it is $G(\bar{\gamma}, Q(\bar{\gamma})) \leq v_{R}-v_{L}<G(\bar{\gamma}, P(\bar{\gamma}))$; and for $\bar{\gamma}>\gamma_{I}$, it is $G\left(\bar{\gamma}, \gamma_{L}\right)<v_{R}-v_{L}<G(\bar{\gamma}, P(\bar{\gamma}))$. Here $\gamma_{I}\left(>\gamma_{m}\right)$ is the strain-level at which the driving force $\hat{f}\left(\gamma_{I}, \gamma_{L}\right)$ vanishes; see Fig. 7. Once ${ }^{+}$has been thus determined, all of the other unknowns can be found in terms of $\bar{\gamma}$ and the initial data without further restriction. Further discussion of this solution is postponed until subsection (iii).

(ii(b)) Solutions with two phase boundaries, one shock wave, and one wave fan. Figure 6(b). Consider next solutions having the form of Fig. 6(b):

$$
\gamma, v= \begin{cases}\gamma_{L}, v_{L}, & -\infty<x<\dot{s}_{1} t, \\ \gamma_{A}, v_{A}, & \dot{s}_{1} t<x<\dot{s}_{2} t, \\ \bar{\gamma}, \bar{v}, & \dot{s}_{2} t<x<\dot{s}_{3} t, \\ \dot{\gamma},+ & \dot{s}_{3} t<x \leq c t \\ \hat{v}, \hat{v}, & \stackrel{c}{c} t \leq x \leq c_{R} t, \\ \gamma_{R}, v_{R}, & c_{R} t \leq x<\infty,\end{cases}
$$

where $\gamma_{A}, v_{A}, \bar{\gamma}, \bar{v}, \stackrel{+}{\gamma}, \stackrel{+}{v}, \dot{s}_{1}, \dot{s}_{2}, \dot{s}_{3}, \stackrel{+}{c}$, and $c_{R}$ are to be determined such that $\gamma_{A}$, 
$\stackrel{+}{\gamma} \in\left(-1, \gamma_{M}\right], \bar{\gamma} \in\left[\gamma_{m}, \infty\right)$, and $\dot{s}_{1}<\dot{s}_{2} \leq 0 \leq \dot{s}_{3}<\stackrel{+}{c} \leq c_{R}$. The functions $\hat{\gamma}, \hat{v}$ correspond to the fields in a low-strain fan and are given by (24)-(25).

The analysis of this case is similar to the previous one and so we merely state the results. Again, there is a two-parameter family of solutions in general, and for algebraic simplicity we assume that $\dot{s}_{3}=-\dot{s}_{2} \equiv \dot{s}$, thus reducing it to a one-parameter family of solutions.

The strains $(\bar{\gamma}, \stackrel{+}{\gamma})$ must lie in the region $D_{2} \subset \Gamma_{3}$ shown in Fig. 7. Given the strain $\bar{\gamma}$, one is to find a root ${ }_{\gamma}^{+}$with $(\bar{\gamma}, \stackrel{+}{\gamma}) \in D_{2}$ of the equation

$$
v_{R}-v_{L}=G(\bar{\gamma}, \stackrel{+}{\gamma})
$$

where $G$ is defined by

$$
\begin{aligned}
G(\bar{\gamma}, \stackrel{+}{\gamma}) \equiv-\sqrt{\left[\hat{\sigma}\left(\gamma_{L}\right)-\hat{\sigma}(\hat{\gamma})\right]\left(\gamma_{L}-\stackrel{+}{\gamma}\right) / \rho}+\int_{\gamma_{R}}^{+} c(\gamma) d \gamma & \text { for }(\bar{\gamma}, \stackrel{+}{\gamma}) \in D_{2} . \\
& +2 \sqrt{[\hat{\sigma}(\bar{\gamma})-\hat{\sigma}(\stackrel{+}{\gamma})]\left(\bar{\gamma}-\stackrel{+}{\gamma}^{+}\right) / \rho}
\end{aligned}
$$

At each fixed $\bar{\gamma}>\gamma_{I}$ the function $G$ increases monotonically with $\stackrel{+}{\gamma}$. Therefore, for each $\bar{\gamma}$ in the respective ranges $\gamma_{I}<\bar{\gamma}<\gamma_{K}$ and $\gamma>\gamma_{K}$, (64) can be uniquely solved for ${ }^{+}$provided $G(\bar{\gamma}, Q(\bar{\gamma})) \leq v_{R}-v_{L}<G\left(\bar{\gamma}, \gamma_{L}\right)$ and $G\left(\bar{\gamma}, \gamma_{R}\right)<v_{R}-v_{L}<$ $G\left(\bar{\gamma}, \gamma_{L}\right)$, respectively. Here $\gamma_{K}\left(>\gamma_{m}\right)$ is the value of strain at which the driving force $\hat{f}\left(\gamma_{K}, \gamma_{R}\right)$ vanishes; see Fig. 7. Once $\stackrel{+}{\gamma}$ has been thus determined, all of the other unknowns can be found in terms of $\bar{\gamma}$ and the given initial data without further restriction. Further discussion of this solution is postponed until subsection (iii).

(ii(c)) Solutions with two phase boundaries and two shock waves. Figure 6(c). Here we seek solutions in the form of Fig. 6(c):

$$
\gamma, v= \begin{cases}\gamma_{L}, v_{L}, & -\infty<x<\dot{s}_{1} t, \\ \gamma_{A}, v_{A}, & \dot{s}_{1} t<x<\dot{s}_{2} t, \\ \bar{\gamma}, \bar{v}, & \dot{s}_{2} t<x<\dot{s}_{3} t, \\ \dot{\gamma},+ & \dot{s}_{3} t<x<\dot{s}_{4} t, \\ \gamma_{R}, v_{R}, & \dot{s}_{4} t<x<\infty,\end{cases}
$$

in which $\gamma_{A}, v_{A}, \bar{\gamma}, \bar{v}, \stackrel{+}{\gamma}, \stackrel{+}{v}, \dot{s}_{1}, \dot{s}_{2}, \dot{s}_{3}, \dot{s}_{4}$ are to be determined such that $\gamma_{A}, \stackrel{+}{\gamma} \in$ $\left(-1, \gamma_{M}\right], \bar{\gamma} \in\left[\gamma_{m}, \infty\right)$, and $\dot{s}_{1}<\dot{s}_{2} \leq 0 \leq \dot{s}_{3}<\dot{s}_{4}$.

The analysis of this case is again similar to the previous ones. There is again a two-parameter family of solutions in general, and for algebraic simplicity we assume that $\dot{s}_{3}=-\dot{s}_{2} \equiv \dot{s}$, thus reducing it to a one-parameter family of solutions.

The strains $(\bar{\gamma}, \stackrel{+}{\gamma})$ must lie in the region $D_{3} \subset \Gamma_{3}$ shown in Fig. 7. Given the strain $\bar{\gamma}$, one is to find a root $\stackrel{+}{\gamma}$ with $(\bar{\gamma}, \stackrel{+}{\gamma}) \in D_{3}$ of the equation

$$
v_{R}-v_{L}=G(\bar{\gamma}, \stackrel{+}{\gamma})
$$


where $G$ is defined by

$$
\begin{aligned}
& G(\bar{\gamma}, \stackrel{+}{\gamma})=-\sqrt{\left[\hat{\sigma}\left(\gamma_{L}\right)-\hat{\sigma}(\stackrel{+}{\gamma})\right]\left(\gamma_{L}-\stackrel{+}{\gamma}\right) / \rho}-\sqrt{\left[\hat{\sigma}\left(\gamma_{R}\right)-\hat{\sigma}(\stackrel{+}{\gamma})\right]\left(\gamma_{R}-\stackrel{+}{\gamma}\right) / \rho} \\
&+2 \sqrt{[\hat{\sigma}(\bar{\gamma})-\hat{\sigma}(\stackrel{+}{\gamma})](\bar{\gamma}-\stackrel{+}{\gamma}) / \rho} \text { for }(\bar{\gamma}, \stackrel{+}{\gamma}) \in D_{3} .
\end{aligned}
$$

At each fixed $\bar{\gamma}>\gamma_{K}$ the function $G$ increases monotonically with increasing $\dot{\gamma}$, and therefore (67) can be uniquely solved for $\stackrel{+}{\gamma}$ provided $G(\bar{\gamma}, Q(\bar{\gamma})) \leq v_{R}-v_{L}<$ $G\left(\bar{\gamma}, \gamma_{R}\right)$. Once $\dot{\gamma}$ has been determined, all of the other unknowns can be found without further restriction.

(ii(d)) Solutions with two phase boundaries, one fan, and one shock wave. Figure 6(d). One can show that solutions having the form shown in Fig. 6(d) do not exist for the initial data (21), (36) being studied here. Such solutions do exist in the case $\gamma_{R}>\gamma_{L}$, in which event solutions of the form of Fig. 6(b) do not exist.

It is clear from (62), (65), and (68) that $G$ is continuous across the lines $I J$ and $K L$ in the $(\bar{\gamma}, \stackrel{+}{\gamma})$-plane shown in Fig. 7. In the respective limits, for $\bar{\gamma}>$ $\gamma_{I}, v_{R}-v_{L} \rightarrow G\left(\bar{\gamma}, \gamma_{L}-\right)$ and $v_{R}-v_{L} \rightarrow G\left(\bar{\gamma}, \gamma_{L}+\right)$, the solution forms shown in Fig. 6(a) and Fig. 6(b) coincide. This limiting solution involves a wave fan traveling right and two phase boundaries. Similarly, the solution forms in Fig. 6(b) and Fig. 6(c) coincide in the limits $v_{R}-v_{L} \rightarrow G\left(\bar{\gamma}, \gamma_{R}-\right)$ and $v_{R}-v_{L} \rightarrow G\left(\bar{\gamma}, \gamma_{R}+\right)$. In this case, the solution involves a leftward moving shock wave and again two phase boundaries.

7. Summary of all solutions. Nonuniqueness. In the preceding section we constructed all solutions to a Riemann problem, and it is useful to examine these solutions on the $\left(\bar{\gamma}, v_{R}-v_{L}\right)$-plane. Note that $\bar{\gamma}$ is the final strain in the bar at large time while $v_{R}-v_{L}$ is part of the given initial data. For solutions that involve a phase change, we map the regions $D_{1}, D_{2}$, and $D_{3}$ of the $(\bar{\gamma}, \stackrel{+}{\gamma})$-plane into the respective regions $D_{1}^{\prime}, D_{2}^{\prime}$, and $D_{3}^{\prime}$ in the $\left(\bar{\gamma}, v_{R}-v_{L}\right)$-plane by using the mapping $v_{R}-v_{L}=G(\bar{\gamma}, \hat{\gamma}), \bar{\gamma}=\bar{\gamma}$. The resulting regions are shown hatched in Fig. 8. The boundary curves $A^{\prime} D^{\prime}, D^{\prime} C^{\prime}, A^{\prime} B^{\prime}, I^{\prime} J^{\prime}$, and $K^{\prime} L^{\prime}$ are the images of the respective curves $A D, D C, A B, I J$, and $K L$, and are defined by $v_{R}-v_{L}=$ $G(\bar{\gamma}, T(\bar{\gamma})), v_{R}-v_{L}=G(\bar{\gamma}, P(\bar{\gamma})), v_{R}-v_{L}=G(\bar{\gamma}, Q(\bar{\gamma})), v_{R}-v_{L}=G\left(\bar{\gamma}, \gamma_{L}\right)$, and $v_{R}-v_{L}=G\left(\bar{\gamma}, \gamma_{R}\right)$, respectively.

The solution corresponding to a point in $D_{1}^{\prime}$ involves two phase boundaries and two wave fans (Fig. 6(a)). At a point in $D_{2}^{\prime}$ the solution involves two phase boundaries, one wave fan, and one shock wave (Fig. 6(b)), while in $D_{3}^{\prime}$ it involves two phase boundaries and two shock waves (Fig. 6(c)). It is useful to note that the solution corresponding to a point on the respective curves $A^{\prime} D^{\prime}, D^{\prime} C^{\prime}$, and $A^{\prime} B^{\prime}$ involves phase boundaries that are stationary, sonic, and dissipation-free, respectively.

Solutions that do not involve a phase change can also be described on this plane by plotting, for each type of solution, the curve $v_{R}-v_{L}=H(\bar{\gamma})$. The result is the curve $P^{\prime} Q^{\prime} R^{\prime} S^{\prime}$ shown in Fig. 8. 


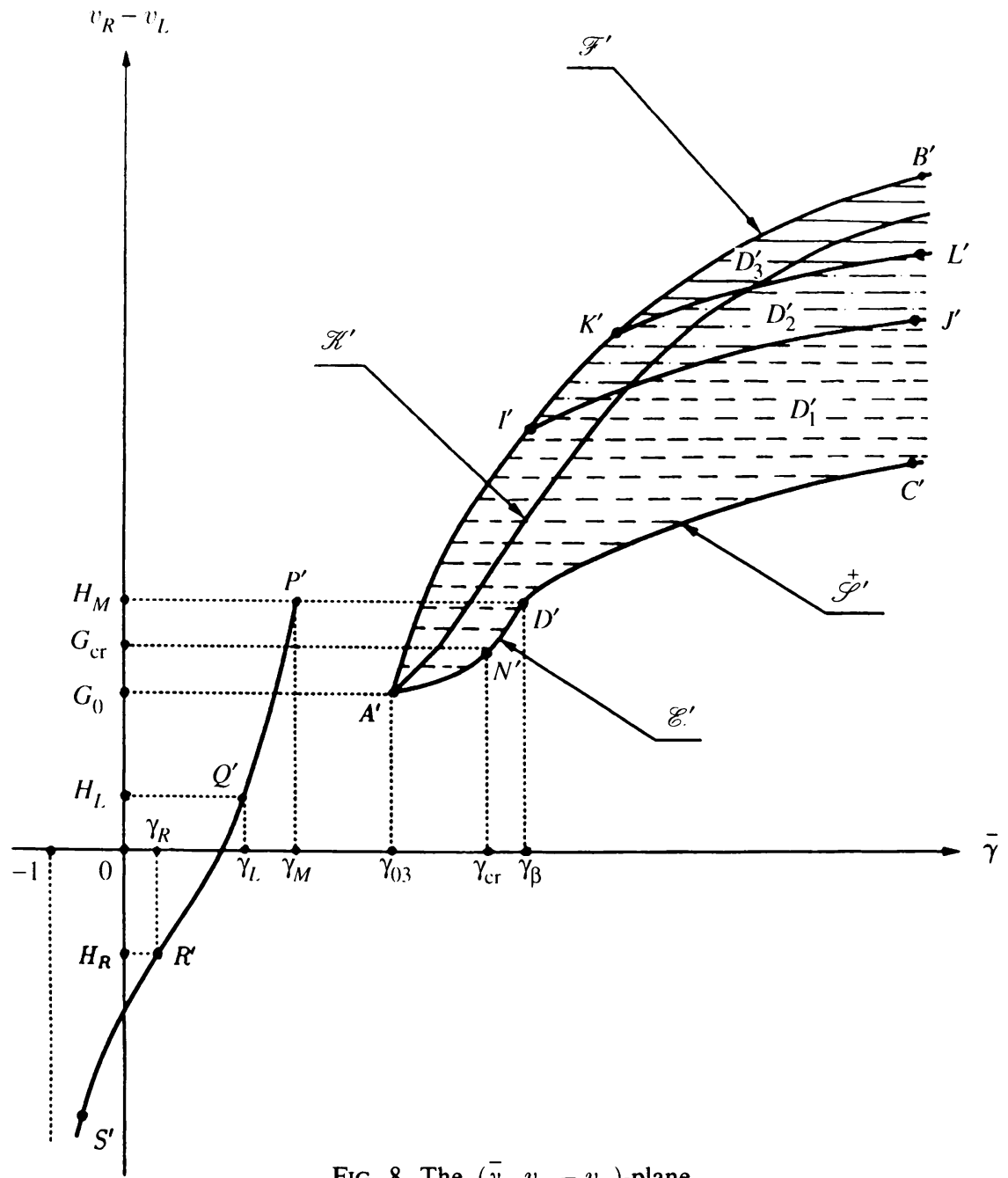

FIG. 8. The $\left(\bar{\gamma}, v_{R}-v_{L}\right)$-plane

Each of the curves $P^{\prime} Q^{\prime} R^{\prime} S^{\prime}, A^{\prime} B^{\prime}, A^{\prime} D^{\prime}, D^{\prime} C^{\prime}, I^{\prime} J^{\prime}$, and $K^{\prime} L^{\prime}$ can be shown to be monotonically increasing. The figure reveals two distinct types of nonuniqueness. For all initial data such that $v_{R}-v_{L}<H_{M}$ there is a unique admissible solution that involves no phase change. On the other hand, when the initial data is such that $v_{R}-v_{L}>G_{0} \equiv G\left(\gamma_{01}, \gamma_{03}\right)$, there is a one-parameter family of solutions all of which involve a phase change; the lack of uniqueness here arises because of the undetermined velocities of the phase boundaries. For initial data on the intermediate interval $H_{M}>v_{R}-v_{L}>G_{0}$, both of these types of solutions are available, in one of which the bar ultimately changes phase, while in the other it does not.

8. Kinetics and nucleation. Uniqueness. The propagation of a phase boundary is controlled by a kinetic relation which, in the simplest models, is a constitutively prescribed relation between the driving force $f$ acting on the phase boundary and 
its propagation speed $\dot{s}$ :

$$
f(t)=\varphi(\dot{s}(t)) .
$$

Continuum theory does not provide an explicit expression for $\varphi$; this is obtained through suitable micro-mechanical modeling. It is sufficient for our purposes to merely assume that $\varphi$ is a continuous function on $\left(-c_{\infty}, c_{\infty}\right)$ that increases with $\dot{s}$ and that the graph of $f=\varphi(\dot{s})$ is a curve that lies in the region $\Gamma_{2}^{\prime} \cup \Gamma_{3}^{\prime}$ described previously of the $(\dot{s}, f)$-plane; see Fig. 3 . The entropy inequality (31) implies that $\varphi(\dot{s}) \dot{s} \geq 0$ and, assuming $\varphi$ to be continuous, that $\varphi(0)=0$.

The kinetic relation controls the propagation of an existing phase boundary. A nucleation criterion is needed in order to signal the onset of a phase transformation when the bar involves only a single phase. We assume that the transformation from the low-strain phase to the high-strain phase is nucleated whenever there exists a solution, corresponding to the given data, with the associated driving force $f$ at least as great as a given critical value $f_{\mathrm{cr}}$; the parameter $f_{\mathrm{cr}}>0$ is also determined by the constitutive details of the material. Once the nucleation criterion indicates that we should consider solutions that do change phase, the kinetic relation selects the particular one of these solutions that is appropriate.

The nucleation criterion $f(\bar{\gamma}, \stackrel{+}{\gamma})=f_{\text {cr }}$ describes a curve $\mathcal{N}$ in the $(\bar{\gamma}, \stackrel{+}{\gamma})$-plane that lies in the hatched portion $\Gamma_{3}$ in Fig. 7. For clarity of the figure, this curve shall not be displayed; it can be readily verified that it has one end at a point $N$ on $A D$, and declines monotonically as $\bar{\gamma}$ increases to $\hat{\gamma} \rightarrow R_{\infty}$ as $\bar{\gamma} \rightarrow \infty$. The coordinates of the point $N$ are found by solving the pair of equations

$$
f(\bar{\gamma}, \stackrel{+}{\gamma})=f_{\mathrm{cr}}, \quad \hat{\sigma}(\bar{\gamma})=\hat{\sigma}(\stackrel{+}{\gamma}) ;
$$

let $\left(\bar{\gamma}_{\mathrm{cr}}, \stackrel{+}{\mathrm{cr}}_{\mathrm{r}}\right)$ be this point and let $G_{\mathrm{cr}} \equiv G\left(\bar{\gamma}_{\mathrm{cr}}, \stackrel{+}{\mathrm{cr}}\right)$ be the corresponding value of $G$ at this point. The image $\mathscr{N}^{\prime}$ of the curve $\mathscr{N}$ in the $\left(v_{R}-v_{L}, \bar{\gamma}\right)$-plane is a curve in the hatched region shown in Fig. 8 that rises monotonically commencing at a point $N^{\prime}$ on $A^{\prime} D^{\prime}$. Again, for clarity of the figure, this image $\mathscr{N}^{\prime}$ shall not be displayed. The ordinate of the point $N^{\prime}$ is $G_{\text {cr }}$. Thus according to the nucleation criterion, a solution with a phase change is selected whenever $v_{R}-v_{L} \geq G_{\mathrm{cr}}$, a solution without phase change is selected whenever $v_{R}-v_{L}<G_{\mathrm{cr}}$.

For $v_{R}-v_{L} \geq G_{\mathrm{cr}}$, we must consider the one-parameter family of solutions that involve a phase change. In order to select the relevant solution from among this family, we begin by applying the kinetic relation (69) to the phase boundaries $x=\dot{s} t$ in each of the solutions in Figs. 6(a), (b), and (c); the kinetic relation at the other phase boundary $x=-\dot{s} t$ holds automatically. Using (7) and (14) in the kinetic relation (69) and ensuring that $\dot{s} \geq 0$ leads to

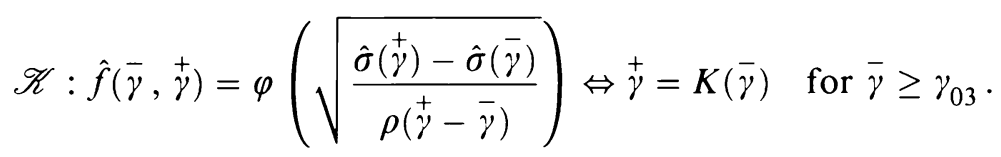

This describes a curve $\mathscr{K}$ in the $(\bar{\gamma}, \stackrel{+}{\gamma})$-plane that lies in the region $\Gamma_{3}$ (the union of the hatched portions in Fig. 7); it commences at the point $A$ and declines monoton- 
ically with $K(\bar{\gamma}) \rightarrow R_{\infty}$ as $\bar{\gamma} \rightarrow \infty$. When mapped from here to the $\left(v_{R}-v_{L}, \bar{\gamma}\right)$ plane, this yields the curve $\mathscr{K}^{\prime}$ defined by $v_{R}-v_{L}=G(\bar{\gamma}, K(\bar{\gamma})), \bar{\gamma} \geq \gamma_{03}$; see Fig. 8. This curve commences at the point $A^{\prime}$ and the monotonicity of the kinetic response function $\varphi$ ensures that it rises monotonically without bound.

Thus since $\mathscr{K}^{\prime}$ increases monotonically for any given $v_{R}-v_{L}>G_{\mathrm{cr}}$, there is a unique strain $\bar{\gamma} \quad\left(>\bar{\gamma}_{\mathrm{cr}}\right)$ that satisfies the kinetic relation.

Thus, in summary, we have shown that for the metastable initial data (21), (36), the Riemann problem has a unique solution that is consistent with the kinetic relation and the nucleation criterion. For initial data such that $v_{R}-v_{L}<G_{\mathrm{cr}}$ this solution involves no phase change and has the form given by Fig. 5; the bar commences in the low-strain phase and remains there for all time. For $v_{R}-v_{L} \geq G_{\mathrm{cr}}$ the solution involves a phase change and has the form given by Fig. 6; in this case the bar, which was initially in the low-strain phase, transforms eventually to the high-strain phase. In either case, the eventual strain in the bar is $\bar{\gamma}$ and can be read from Fig. 8 .

In closing, we note that some solutions to the present problem involved phase boundaries that were subsonic while others involved sonic phase boundaries (the latter correspond to points on $D^{\prime} C^{\prime}$ in Fig. 8). In the present analysis the kinetic relation was imposed on both of these types of phase boundaries and this leads to uniqueness. If the kinetic law had been applied only to subsonic phase boundaries, then solutions corresponding to points on both curves $\mathscr{K}^{\prime}$ and $D^{\prime} C^{\prime}$ would be allowed. In this case the Riemann problem corresponding to given data would have a unique solution for $v_{R}-v_{L}<H_{M}$ (see Fig. 8) but for $v_{R}-v_{L} \geq H_{M}$ the problem would have two solutions, one satisfying the kinetic relation and the other involving a sonic phase boundary. This was first observed, in a particular setting, by Truskinovsky [18]; he takes the view that the kinetic relation should only be applied to subsonic phase boundaries and suggests that the accompanying nonuniqueness (for $v_{R}-v_{L} \geq H_{M}$ in the present setting) describes an instability.

Acknowledgment. I would like to express my appreciation to my thesis supervisor, Professor Rohan Abeyaratne, for his constant support, valuable guidance, and technical assistance during the whole course of this work. I am grateful to Professor Lev Truskinovsky for providing us with a preprint of his study [18]. This work was supported by O.N.R. N00014-90-J-1871 and A.R.O. DAAL 03-91-G-0011 to whom we express our thanks.

\section{REFERENCES}

[1] R. Abeyaratne, Discontinuous deformation gradients in the finite twisting of an elastic tube, Journal of Elasticity 11, 43-80 (1981)

[2] R. Abeyaratne and J. K. Knowles, On dissipative response due to discontinuous strains in bars of unstable elastic material, Internat. J. Solids Structures 24, 1021-1024 (1988)

[3] R. Abeyaratne and J. K. Knowles, Kinetic relations and the propagation of phase boundaries in solids, Arch. Rational Mech. Anal. 114 (1991)

[4] R. Abeyaratne and J. K. Knowles, Implications of viscosity and strain gradient effects for kinetics of propagating phase boundaries in solids, SIAM (to appear)

[5] R. Abeyaratne and J. K. Knowles, On the propagation of maximally dissipative phase boundaries in solids, Quart. Appl. Math. 50, 149-172 (1992) 
[6] J. M. Ball and R. D. James, Fine phase mixtures as minimizers of energy, Arch. Rational Mech. Anal. 100, 13-53 (1987)

[7] J. W. Christian, The theory of transformations in metals and alloys, Part I, Purgations, Oxford, 1975

[8] C. M. Dafermos, The entropy rate admissibility criterion for solutions of hyperbolic conservation laws, J. Differential Equations 14, 202-212 (1973)

[9] C. M. Dafermos, Discontinuous thermokinetic process, Appendix 4B of Rational Thermodynamics, by C. Truesdell, Springer-Verlag, New York, 1984

[10] J. L. Ericksen, Equilibrium of bars, Journal of Elasticity 5, 191-201 (1975)

[11] H. Hattori, The Riemann problem for a van der Waals fluid with entropy admissibility criterion. Nonisothermal case, J. Differential Equations 65, 158-174 (1986)

[12] R. D. James, The propagation of phase boundaries in elastic bars, Arch. Rational Mech. Anal. 73, 125-158 (1980)

[13] T. P. Liu, Uniqueness of weak solutions of the Cauchy problem for general $2 \times 2$ conservation laws, J. Differential Equations 20, 369-388 (1976)

[14] O. A. Oleinik, On the uniqueness of the generalized solution of the Cauchy problem for nonlinear system of equations occurring in mechanics, Uspekhi Matematicheskii Nauk (N.S.) 126 (78), 169-176 (1957) (Russian)

[15] M. Slemrod, Admissibility criterion for propagating phase boundaries in a van der Waals fluid, Arch. Rational Mech. Anal. 81, 301-315 (1983)

[16] M. Shearer, Nonuniqueness of admissible solutions of Riemann initial value problems for a system of conservation laws of mixed type, Arch. Rational. Mech. Anal. 93, 45-69 (1986)

[17] L. Truskinovsky, Equilibrium phase interface, Soviet Physics Doklady 27, 551-553 (1982)

[18] L. Truskinovsky, Transition to "detonation" in dynamic phase changes, preprint, September, 1992

[19] Y. Lin, Ph.D. thesis, Massachusetts Institute of Technology, Cambridge, MA, 1993 